\title{
Article \\ Rice (Oryza sativa) TIR1 and 5'adamantyl-IAA Significantly Improve the Auxin-Inducible Degron System in Schizosaccharomyces pombe
}

\author{
Adam T. Watson ${ }^{1}\left(\mathbb{D}\right.$, Storm Hassell-Hart ${ }^{2}$, John Spencer ${ }^{2} \mathbb{D}$ and Antony M. Carr ${ }^{1, *(1)}$ \\ 1 Genome Damage and Stability Centre, School of Life Sciences, University of Sussex, Brighton BN1 9RQ, UK; \\ a.t.watson@sussex.ac.uk \\ 2 Department of Chemistry, School of Life Sciences, University of Sussex, Brighton BN1 9QJ, UK; \\ S.Hassell-Hart@sussex.ac.uk (S.H.-H.); J.Spencer@sussex.ac.uk (J.S.) \\ * Correspondence: a.m.carr@sussex.ac.uk
}

check for updates

Citation: Watson, A.T.; Hassell-Hart, S.; Spencer, J.; Carr, A.M. Rice (Oryza sativa) TIR1 and 5 'adamantyl-IAA Significantly Improve the

Auxin-Inducible Degron System in Schizosaccharomyces pombe. Genes 2021, 12, 882. https://doi.org/10.3390/ genes12060882

Academic Editors: Martin Kupiec and Maciej Wnuk

Received: 6 May 2021

Accepted: 3 June 2021

Published: 8 June 2021

Publisher's Note: MDPI stays neutral with regard to jurisdictional claims in published maps and institutional affiliations.

Copyright: (c) 2021 by the authors. Licensee MDPI, Basel, Switzerland. This article is an open access article distributed under the terms and conditions of the Creative Commons Attribution (CC BY) license (https:// creativecommons.org/licenses/by/ $4.0 /)$.

\begin{abstract}
The auxin-inducible degron (AID) system is a powerful tool to induce targeted degradation of proteins in eukaryotic model organisms. The efficiency of the existing Schizosaccharomyces pombe AID system is limited due to the fusion of the F-box protein TIR1 protein to the SCF component, Skp1 (Skp1-TIR1). Here, we report an improved AID system for S. pombe that uses the TIR1 from Oryza sativa (OsTIR1) not fused to Skp1. Furthermore, we demonstrate that degradation efficiency can be improved by pairing an OsTIR1 auxin-binding site mutant, OsTIR1 ${ }^{\text {F74A }}$, with an auxin analogue, 5'adamantyl-IAA (AID2). We provide evidence for the enhanced functionality of the OsTIR1 AID and AID2 systems by application to the essential DNA replication factor Mcm4 and to a non-essential recombination protein, Rad52. Unlike AID, no detectable auxin-independent depletion of AID-tagged proteins was observed using AID2.
\end{abstract}

Keywords: AID; AID2; degron; auxin; fission yeast; protein degradation

\section{Introduction}

The auxin-inducible degron (AID) is an effective method for the rapid depletion of target proteins, allowing control of protein expression and the study of protein function in vivo [1]. In plants, the physiological role of auxin is to regulate growth and development by mediating the interaction of the F-box protein transport inhibitor response 1 (TIR1) with the auxin/indole-3-acetic acid (AUX/IAA) family of transcriptional regulators [2]. TIR1 is part of the E3 ubiquitin ligase SCF-TIR1 (Skp/Cullin/F-box) complex that recruits an E2 ubiquitin-conjugating enzyme and polyubiquitinates AUX/IAA proteins, targeting them for degradation by the proteasome.

This degradation pathway can be transferred to non-plant organisms by (1) exogenously expressing the auxin receptor F-box protein, TIR1 and (2) the fusion of an AUX/IAA auxin-inducible degron (AID) to the protein of interest. As the other components of the SCF complex are highly conserved, these non-plant cells can be treated with auxin to induce proteasomal degradation of the AID-tagged protein (Figure 1A). In current AID systems, Oryza sativa TIR1 (OsTIR1) is the most commonly used auxin receptor F-box protein and is used in combination with AID tags derived from the Arabidopsis thaliana IAA17 protein [1,3-9]. However, the fission yeast AID system developed by Kanke et al. [10] uses a fusion of the A. thaliana TIR1 (AtTIR1) to the F-box-interacting component of the SCF, Skp1 (Skp1-AtTIR1, system termed ' $i$-AID'). Overexpression of the Skp1-AtTIR1 fusion using the constitutive alcohol dehydrogenase $a d h 1$ promoter severely inhibited cell growth, so expression levels were reduced using an attenuated version of the adh1 promoter, Padh15 (Padh15-skp1-AtTIR1) [11]. Despite $i$-AID causing severe replication defects and cell cycle arrest in mcm4-AID cells, biologically significant amounts of other target proteins were 
shown to remain after depletion. The system was improved by exogenous expression of Padh15-skp1-AtTIR1 and Padh15-skp1-OsTIR1 (termed 'double TIR1'). Double TIR1 combined with transcription repression of the target protein (termed 'off-AID') was used to effectively deplete Pol1 and Cdc45 [10]. The off-AID system has been used to efficiently regulate various other proteins including Mcm10 [12], Cdc20 [13], Cnd3 and Smc2 [14] and Bqt1 [15].

A common feature of AID systems is the chronic, auxin-independent proteasomemediated degradation of AID-tagged proteins in cells constitutively expressing TIR1. To circumvent this basal degradation level and its inherent problems, expression of TIR1 is often transcriptionally regulated. Alternatively, another component of the plants native auxin signalling machinery, an auxin-response transcription factor (ARF), is co-expressed as this has been shown to allow near-endogenous expression levels of the target protein in the absence of auxin [16].

Through a bump-and-hole strategy, a synthetic auxin-receptor pair involving 5-(3methoxy-phenyl)-IAA and its complementary auxin-binding pocket mutant AtTIR1 ${ }^{\mathrm{F} 79 \mathrm{G}}$ was identified and shown to function orthogonally to endogenous auxin signalling [17]. A screen of 5-substituted IAA analogues using a yeast two-hybrid system identified 5'adamantylindole-3-acetic acid ( $5^{\prime}$ adamantyl-IAA) which mediates interaction of AtTIR1 ${ }^{\mathrm{F} 79 \mathrm{G}}$ and IAA3 proteins at a 100-fold lower concentration compared to the natural auxin-TIR1 pair. Following a screen of binding pocket mutants other than glycine, AtTIR $1^{\mathrm{F} 79 \mathrm{~A}}$ was shown to interact with IAA3 proteins at picomolar concentrations of $5^{\prime}$ adamantyl-IAA, 10,000-fold lower than for the auxin-TIR1 pair [18]. The corresponding OsTIR1-binding site mu$\operatorname{tant}\left(\mathrm{OsTIR} 1^{\mathrm{F} 74 \mathrm{~A}}\right)$ and $5^{\prime}$ adamantyl-IAA have recently been shown to function in chicken DT40 cells at 1,000-fold lower ligand concentration compared to conventional AID/IAAOsTIR $1^{\text {WT }}$ and shown to function effectively in mammalian cell lines again at significantly lower ligand concentrations [19]. Using an equivalent bump-and-hole strategy, the synthetic ligand-receptor pair OsTIR1 ${ }^{\mathrm{F} 74 \mathrm{G}}$ and auxin analogue 5-phenyl-indole-3-acetic acid (5-Ph-IAA) were shown to interact at nanomolar concentrations and have been used to rapidly and efficiently deplete mAID-fused proteins in budding yeast, mammalian cells and mice. This modified system was termed AID version 2 (AID2) [6]. The kinetics of protein depletion were quicker when compared to the original AID system and depletion could be induced with several hundred-fold lower ligand concentration. Moreover, no detectable basal degradation was observed using AID2.

In fission yeast, a mechanism for the selective removal of meiosis-specific mRNAs in mitotic cells has been characterised. The removal involves the YTH domain-containing protein Mmi1 [20], which binds meiosis-specific mRNAs containing Determinant of Selective Removal (DSR) sequences, usually located at the $3^{\prime}$ end of the transcript. Mmi1 is only functional in mitotic, but not meiotic, cells and greatly increases transcript turnover by directing DSR-containing transcripts to the nuclear exosomes for degradation [20,21]. By deletion analysis, the DSR elements of $s s m 4$, rec 8 and spo5 mRNAs were identified [20]. Further studies have identified a hexanucleotide motif, U(U/C)AAAC, that is highly enriched in the DSR elements and have shown that tandem repeats of this motif can function as an artificial DSR in heterologous gene systems $[22,23]$. 
(A) AID

(B)

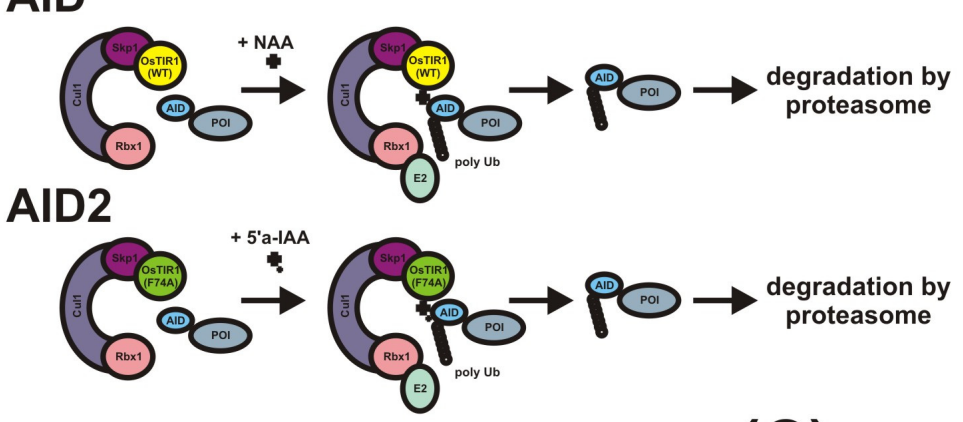

AID2

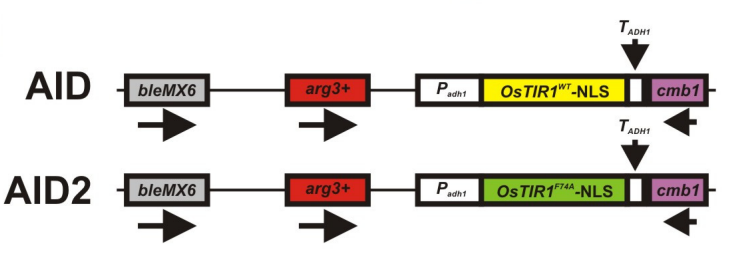

(C)

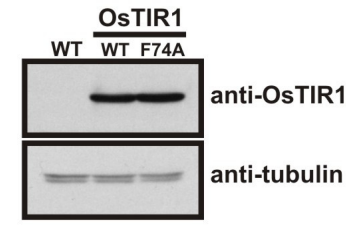

(D)

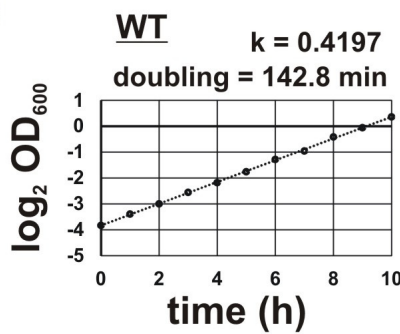

(E)
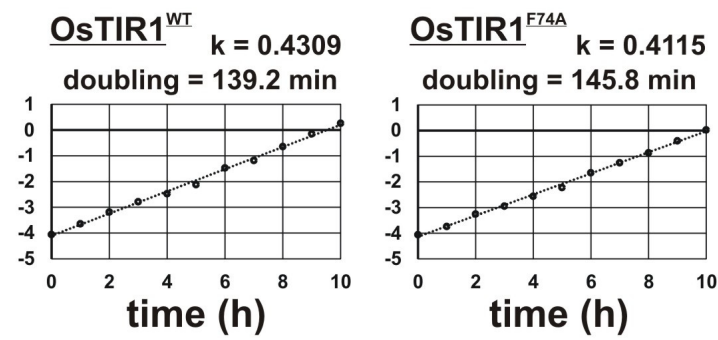

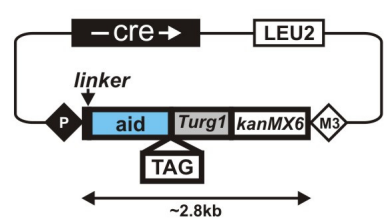

pAW8-aid-Turg1-kanMX6 (169354)

pAW8-aid-3HA-Turg1-kanMX6 (169355)

pAW8-aid-5myc-Turg1-kanMX6 (169356)

pAW8-aid-V5-Turg1-kanMX6 (169357)

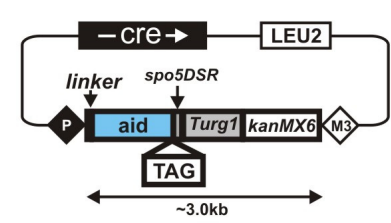

pAW8-aid-spo5DSR-Turg1-kanMX6 (169358)

pAW8-aid-3HA-spo5DSR-Turg1-kanMX6 (169359)

pAW8-aid-5myc-spo5DSR-Turg1-kanMX6 (169360)

pAW8-aid-V5-spo5DSR-Turg1-kanMX6 (169361)

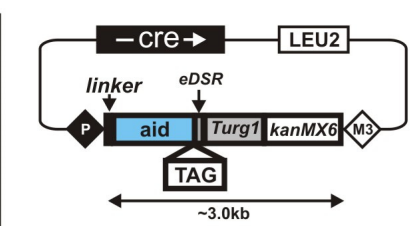

pAW8-aid-eDSR-Turg1-kanMX6 (169362)

pAW8-aid-3HA-eDSR-Turg1-kanMX6 (169363)

pAW8-aid-5myc-eDSR-Turg1-kanMX6 (169364)

(F)

forward primer: 5' - (gene specific sequence) - GGT GGG TCA GGT GGA AGT G - 3"*

reverse primer: 5' - (gene specific sequence) - GGATGGCGGCGTTAGTATC - 3'
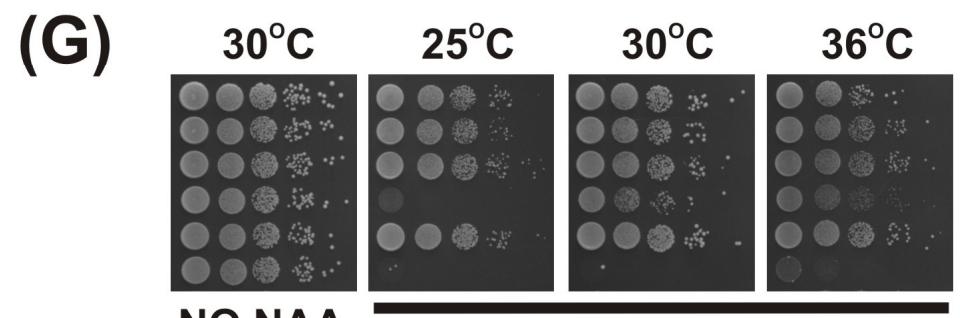

mcm4+

mcm4-AID

mcm4+ $\rceil+$ Skp1-AtTIR1

mcm4-AID

mcm4+

mcm4-AID +OSTIR1 ${ }^{w T}$

NO NAA

\section{$500 \mu \mathrm{M}$ NAA}

Figure 1. AID/OsTIR1 ${ }^{\mathrm{WT}}$ and AID2/OsTIR1 ${ }^{\mathrm{F} 74 \mathrm{~A}}$ systems in S. pombe. (A) Schematic illustration showing AID/OsTIR1 ${ }^{\mathrm{WT}}$ and AID2/OsTIR1 ${ }^{\text {F74A }}$ systems. AID: As the Skp1, Cullin and F-box protein complex (SCF) is highly conserved among eukaryotes, exogenously expressed plant F-box protein OsTIR1 can form a functional SCF complex in S. pombe. The protein of interest (POI) is fused to the AID degron (AID). The synthetic auxin 1-naphthaleneacetic acid (NAA) binds OsTIR1 and 
promotes interaction between OsTIR1 and the AID degron. SCF-OsTIR1 acts as an E3 ubiquitin ligase to recruit an E2 ubiquitin-conjugating enzyme, resulting in the poly-ubiquitination of the AID tag. Finally, the POI is degraded by the proteasome. AID2: The process is analogous to AID, but pairs the OsTIR1 auxin-binding site mutant F74A and the 5'-substituted auxin analogue 5' adamantyl-indole-3-acetic acid (5'a-IAA). (B) Schematic illustration showing the arg3-D4 loci engineered to constitutively express OsTIR1 ${ }^{\mathrm{WT}}$ or OsTIR1 ${ }^{\mathrm{F} 74 \mathrm{~A}}$ (NLS: nuclear localisation signal). (C) Representative immunoblot on total protein extract from wild-type ${ }^{\text {WT }}$ (AW279), OsTIR1 ${ }^{\text {WT }}$ (AW1762) and OsTIR1 ${ }^{\text {F74A }}$ (AW1703) cells detected using anti-OsTIR1 antibody. Anti-tubulin was used as a loading control. (D) Representative growth curves of isogenic wild-type (WT-(AW279)), OsTIR1 ${ }^{W T}$ (AW1762) and OsTIR1 ${ }^{F 74 A}$ (AW1703) cells. Cells were grown in YE media at $30{ }^{\circ} \mathrm{C}$ with constant shaking (180 rpm). Optical density (OD) was measured at $1 \mathrm{~h}$ intervals for total $10 \mathrm{~h}$. Timeseries of $\log$ 2-transformed $\mathrm{OD}_{600}$ measurements are presented. Black lines represent linear regression models and are the mean of two technical repeats. Slopes of linear regression models $(k)$ and calculated doubling times are indicated. (E) Plasmids to C-terminally AID-tag a protein of interest (linker: flexible 10 amino acid linker (Gly-Gly-Ser-Gly-Gly-Ser-Gly-Ser-Gly-Ala), spo5DSR: spo5 Determinant of Selective Removal element, eDSR: enhanced spo5DSR element, TAG: epitope tag, number in parenthesis after plasmid name: Addgene ID). (F) Primer sequences to amplify aid-(TAG)-(DSR)-Turg1-kanMX6 construct. * Reading frame for tag is indicated. (G) Isogenic strains with the indicated genotypes (mcm4+ (AW279), mcm4-AID (AW1893), Skp1-AtTIR1 (AW1942), mcm4-AID Skp1-AtTIR1 (AW1949), OsTIR1 ${ }^{W T}$ (AW1762), mcm4-AID, OsTIR1 ${ }^{\text {WT }}$ (AW1923)) were spotted onto YEA plates and plates containing $500 \mu \mathrm{M}$ NAA. Plates were grown at the indicated temperatures for 3 days. The experiment was repeated and similar results were obtained.

Here, we describe an improved AID system for fission yeast that overcomes the drawbacks of the original Kanke et al. [10] approach. We constructed two sets of plasmid vector for this study. One set was designed to stably integrate expression constructs at the arg3-D4 locus [24]. These plasmids were used to insert and constitutively express a genomic copy of the OsTIR1 receptor, OsTIR1 ${ }^{\mathrm{WT}}$, or the AID2 variant, OsTIR1 ${ }^{\mathrm{F} 74 \mathrm{~A}}$, using the fullstrength $S$. pombe alcohol dehydrogenase (adh1) promoter. The second set of plasmids was designed to allow AID tagging of the protein of interest at the C-terminus. These plasmids include a series of epitope tag sequences for detection of AID-tagged proteins by commercially available antibodies including HA, Myc and V5. The spo5DSR element and an improved engineered version of the spo5DSR element, termed 'eDSR', are also included as an option that will act to constitutively reduce AID-tagged protein levels. The addition of either the synthetic auxin 1-naphthaleneacetic acid (NAA) (OsTIR1 WT / AID) or the 5-substituted auxin 5'adamantyl-IAA (OsTIR1 ${ }^{\text {F74A } / A I D 2), ~ a l l o w e d ~ t h e ~ c r e a t i o n ~ o f ~}$ conditional loss of function mutants of the MCM complex component Mcm4 to be created in cells grown at $30{ }^{\circ} \mathrm{C}$ without fusing to Skp1. For AID2, we observed faster depletion kinetics at lower ligand concentrations as compared to AID and no basal degradation of AID-tagged Mcm4. However, for the non-essential recombination protein Rad52, a complete conditional null phenotype was not obtained, despite the presence of DSR sequences: we observed that very low levels of Rad52 activity remained.

\section{Materials and Methods}

\subsection{Strains and Growth Conditions}

Strains used in this work are listed in Table 1. All strains were grown at $30{ }^{\circ} \mathrm{C}$ unless stated otherwise. The media composition was as described by Moreno et al. [25]. For selection of G418 and phleomycin-resistant cells, G418 disulphate (Formedium, Hunstanton, UK) or phleomycin (Fisher BioReagents, Waltham, MA, USA) was added to YEA plates at a final concentration of 200 and $30 \mu \mathrm{g} / \mathrm{mL}$, respectively. Synthetic plant auxin 1-naphthaleneacetic acid (NAA) (Sigma, St. Louis, MO, USA) powder was dissolved in $80 \%$ ethanol to the required concentration $(0.5 \mathrm{M})$. The final NAA concentration used was $500 \mu \mathrm{M}$. NAA stock was prepared fresh prior to use and unused stock discarded. 5 'adamantyl-IAA was dissolved in DMSO to the required concentration $(1 \mathrm{mM})$ and aliquots stored at $-20^{\circ} \mathrm{C}$. Growth curve doubling times were calculated using the formula $\mathrm{DT}=1 / \mathrm{k}$, where DT stands for doubling time and $\mathrm{k}$ represents the slope of linear regression computed from a timeseries of log 2-transformed OD measurements [26]. 
Table 1. Strains used in this study.

\begin{tabular}{|c|c|}
\hline Name & Genotype ( $h+/ h$ - Denote Mating Type) \\
\hline AW279 & $h+$ \\
\hline AW1502 & $\arg 3-D 4 h+$ \\
\hline AW1581 & rad52::kanMX6 h+ \\
\hline AW1655 & bleMX6-arg3-D4 h- \\
\hline AW1658 & arg3::bleMX6-arg3+-Padh1-OsTIR1 ${ }^{F 74 A}-T A D H 1 ~ h-$ \\
\hline AW1660 & arg3::bleMX6-arg3+-Padh1-OsTIR1 ${ }^{W T}$-TADH1 h- \\
\hline AW1680 & rad52-HA-AID-Turg1:kanMX6, arg3::bleMX6-arg3+-Padh1-OsTIR1 ${ }^{\text {F74A-TADH1 h- }}$ \\
\hline AW1681 & rad52-HA-AID-Turg1:kanMX6, arg3::bleMX6-arg3+-Padh1-OsTIR1 ${ }^{F 74 A}-T A D H 1, h+$ \\
\hline AW1682 & rad52-HA-AID-spo5DSR-Turg1:kanMX6, arg3::bleMX6-arg3+-Padh1-OsTIR1 ${ }^{\text {F74A-TADH1, } h+}$ \\
\hline AW1683 & rad52-HA-AID-spo5DSR-Turg1:kanMX6, arg3::bleMX6-arg3+-Padh1-OsTIR1 ${ }^{F 74 A}-T A D H 1, h-$ \\
\hline AW1703 & arg3::bleMX6-arg3+-Padh1-OsTIR1 ${ }^{\text {F74A-TADH1 h+ }}$ \\
\hline AW1762 & arg3::bleMX6-arg3+-Padh1-OsTIR1 ${ }^{W T}$-TADH1 h+ \\
\hline AW1893 & mcm4-AID-Turg1:kanMX6, h+ \\
\hline AW1899 & mcm4-AID-V5-Turg1:kanMX6, h+ \\
\hline AW1901 & rad52-AID-Turg1:kanMX6, h+ \\
\hline AW1907 & rad52-AID-V5-Turg1:kanMX6, h+ \\
\hline AW1915 & rad52-AID-spo5DSR-Turg1:kanMX6 h+ \\
\hline AW1921 & rad52-AID-V5-spo5DSR-Turg1:kanMX6 h+ \\
\hline AW1923 & mom4-AID-Turg1:kanMX6, arg3::bleMX6-arg3+-Padh1-OsTIR1 ${ }^{\text {WT }}$-TADH1 h+ \\
\hline AW1925 & mcm4-Turg1:kanMX6, arg3::bleMX6-arg3+-Padh1-OsTIR1 F74A_TADH1 h+ \\
\hline AW1935 & mcm4-AID-V5-Turg1:kanMX6, arg3::bleMX6-arg3+-Padh1-OsTIR1 ${ }^{W T}$-TADH1 h+ \\
\hline AW1937 & mom4-AID-V5-Turg1:kanMX6, arg3::bleMX6-arg3+-Padh1-OsTIR1F74A-TADH1 h+ \\
\hline AW1942 & ade6::ade6+-Padh15-skp1-AtTIR1-2NLS-9myc h+ \\
\hline AW1949 & mcm4-AID-Turg1:kanMX6, ade6::ade6+-Padh15-skp1-AtTIR1-2NLS-9myc h+ \\
\hline AW1962 & rad52-AID-Turg1:kanMX6, arg3::bleMX6-arg3+-Padh1-OsTIR1 F74A_TADH1 h+ \\
\hline AW1968 & rad52-AID-V5-Turg1:kanMX6, arg3::bleMX6-arg3+-Padh1-OsTIR1 ${ }^{\text {F74A-TADH1 h+ }}$ \\
\hline AW1970 & rad52-AID-spo5DSR-Turg1:kanMX6, arg3::bleMX6-arg3+-Padh1-OsTIR1 ${ }^{\text {F74A-TADH1 h+ }}$ \\
\hline AW1976 & rad52-AID-V5-spo5DSR-Turg1:kanMX6, arg3::bleMX6-arg3+-Padh1-OsTIR1 ${ }^{\text {F74A-TADH1 h+ }}$ \\
\hline AW1990 & rad52-AID-Turg1:kanMX6, arg3::bleMX6-arg3+-Padh1-OsTIR1 ${ }^{W T}$-TADH1 h+ \\
\hline AW1996 & rad52-AID-V5-Turg1:kanMX6, arg3::bleMX6-arg3+-Padh1-OsTIR1 ${ }^{W T}-T A D H 1 ~ h+$ \\
\hline AW2011 & rad52-AID-eDSR-Turg1:kanMX6 h+ \\
\hline AW2017 & rad52-AID-V5-eDSR-Turg1:kanMX6 h+ \\
\hline AW2024 & rad52-AID-eDSR-Turg1:kanMX6, arg3::bleMX6-arg3+-Padh1-OsTIR1F74A-TADH1 h+ \\
\hline AW2026 & rad52-AID-V5-eDSR-Turg1:kanMX6, arg3::bleMX6-arg3+-Padh1-OsTIR1 F74A-TADH1 h+ \\
\hline
\end{tabular}

\subsection{Cloning and Plasmid Construction}

Q5 High-Fidelity 2X Master Mix was used for all PCR reactions in accordance to the manufacturer's instructions. For all cloning procedures, NEBuilder HiFi DNA Assembly Master Mix was used in accordance to the manufacturer's instructions. Escherichia coli strain $\mathrm{DH} 5 \alpha$ was used for transformations and plasmid isolation. Synthesised gene fragments and custom oligonucleotide primers (Table 2) were ordered from Integrated DNA Technologies. 
Table 2. Sequences of oligonucleotide primers used in this study.

\begin{tabular}{|c|c|}
\hline Name & Sequence $\left(5^{\prime}\right.$ to $\left.3^{\prime}\right)$ \\
\hline P1 & AGAAATCTATAGAAAAAAAGCTAGCGTGACGCAGACA \\
\hline $\mathrm{P} 2$ & CTCCCGGGAGTGCATGCCAGCATATGTATGTGGTTAGAAAAAAGAAAAGACTTAAAAG \\
\hline P3 & AGAAATCTATAGAAAAAAAGCTAGCGTGACGCAGACATTCGAATGGCATGCCC \\
\hline $\mathrm{P} 4$ & $\begin{array}{c}\text { CTCCCGGGAGTGCATGCCAGCATATGTATGTGGTTAGAAAAAAGAAAAGACTT } \\
\text { AAAAGTTTGTGATAGTCAAGACAATGGAATTCTCTTGCTTAAAGAAAAGCGAAGGCA }\end{array}$ \\
\hline P5 & GAGAGCTCCGTCTGCAGCGAGTCGACACTTCTAAATAAGCGAATTTCTTATGATTTATGA \\
\hline P6 & ACACTCTACTTGCCCAGATCACTAGTATATTACCCTGTTATCCCTAGCG \\
\hline P7 & CATACATTATACGAAGTTATGCATGCTCGGTGGGTCAGGTGGAAGTGGATCTGGTGCTATGATGGGCAGTGTCGA \\
\hline P8 & AAATTCGCTTATTTAGAAGTGCTAGCTCAAGCTCTGCTCTTGCACTTC \\
\hline P9 & AGTGCAAGAGCAGAGCTTGAGCTAGCAGTCCCGGGGCTTGCCCATCTGTTTTAGACGT \\
\hline P10 & GGGGACGAGGCAAGCTAAACAGATCTCGAGAAGAAGGCCCCGCTG \\
\hline P11 & AGTGCAAGAGCAGAGCTTGAGCTAGCACTACGCCATATCATGCCCA \\
\hline P12 & TCTAAAACAGATGGGCAAGCCCCGGGGCTTTGTCTAACAGGTTTTATGTTGGTTTAAGT \\
\hline P13 & AGAAGTGCAAGAGCAGAGCTGGTGCTGGAGCAGGCGCCTACCCATACGATGTTCCTGACTATGCG \\
\hline P14 & GATGGGCAAGCCCCGGGACTGCTAGCGGCGCGCCTCAGCACTGAGC \\
\hline P15 & TGGGCATGATATGGCGTAGTGCTAGCGGCGCGCCTCAGCACTGAGC \\
\hline P16 & AGAAGTGCAAGAGCAGAGCTGGAGCAGGCGCCGGTGAACAAAAGTTGATTTCTGAAG \\
\hline P17 & GATGGGCAAGCCCCGGGACTGCTAGCGGCGCGCCTTACAAGTCTTCCTC \\
\hline P18 & TGGGCATGATATGGCGTAGTGCTAGCGGCGCGCCTTACAAGTCTTCCTC \\
\hline P19 & $\begin{array}{l}\text { TATACATTATTTAATACTAGTCCTAACTGACACAGTACAATATTCATT } \\
\text { ATTTCTATGCAAGCCAGTTCAATATCTTGATTGTTTAGCTTGCCTCGTCCCC }\end{array}$ \\
\hline P20 & $\begin{array}{l}\text { TCATCAGGGTCATTGGGACTATTCAACGCGAAATCAAATAGAAAACA } \\
\text { AAATATTGTTTCAAAAGAATGCTTTCATGTATAGGATGGCGGCGTTAGTATCG }\end{array}$ \\
\hline P21 & $\begin{array}{c}\text { GTGCTTTGGAAAGGCGAGGACGTATTAAGGTTATTACCAGTGCT } \\
\text { GGACATCGCATTGTACGTTCAATTGCACAGACTGATGGTGGGTCAGGTGGAAGTG }\end{array}$ \\
\hline P22 & $\begin{array}{l}\text { ATTATGCTCTGTAGTCTTTGATTTTCAACAACGACCTATGGTTTATGG } \\
\text { CTCATGAATAATACCAGCTTATTCGCTAAAAAAGGATGGCGGCGTTAGTATC }\end{array}$ \\
\hline P23 & $\begin{array}{c}\text { GAACAAATTCTGATCCTCAGTCGGCAATGAGGTCGCGAGAAAACTAC } \\
\text { GATGCTACGGTGGATAAGAAAGCCAAAAAAGGAGGTGGGTCAGGTGGAAGTG }\end{array}$ \\
\hline $\mathrm{P} 24$ & $\begin{array}{l}\text { AGATCTACCGTTTAAACAAATCATTAGTCATAAAACAGAAAATACTTGGTAA } \\
\text { AAAACAAGTTGCCAATCATCACATTTTGCCTCATTACTTGGATGGCGGCGTTAGTATC }\end{array}$ \\
\hline
\end{tabular}

\subsection{S. pombe arg3-D4 Integration Expression Vectors}

An NdeI site contained within the pUC19 backbone was removed by restriction with NdeI, blunting the overhangs using Mung Bean Nuclease (New England Biolabs, Ipswich, MA, USA) and religation using T4 DNA ligase (New England Biolabs). The resulting plasmid was termed pUC19*, where * denotes removal of the NdeI recognition site from plasmid backbone (CATATG converted to CATG). The S. pombe arg3-D4 locus was sequenced and the deleted genomic fragment identified. The deleted fragment and approximately $500 \mathrm{bp}$ each side of the deletion site were gene synthesised. Gene synthesis allowed for the removal of unwanted restriction enzymes sites, insertion of a multiple clone site (MCS NheI-NdeI-SphI-XmaI-SacI-PstI-SalI-SpeI) and insertion NotI restriction enzyme sites for the removal of the linear arg3 construct. The gene fragment was cloned into EcoRI/HindIII restricted pUC19* to create MCS- Sp.arg3+/pUC19*. The 820 bp of the S. pombe adh1 promoter sequence (TATA box = TATAAATA) was amplified from total genomic DNA using primers $\mathrm{P} 1$ and $\mathrm{P} 2$ and the resulting fragment cloned into NheI/NdeI restricted 
MCS-Sp.arg3+/pUC19* to create $P_{\text {adh1 }}$-MCS-Sp.arg3+/pUC19*. Approximately $820 \mathrm{bp}$ of the $S$. pombe adh15 (TATA box = TAAATATA) and adh81 (TATA box = AT) promoter sequences were amplified from pKM104 and pKM105 [10] using primers P3 and P4 and the resulting fragment cloned into NheI/NdeI restricted MCS-Sp.arg3+/pUC19* to create $P_{\text {adh } 15}$-MCS-Sp.arg3+/pUC19* and $P_{\text {adh } 81}$-MCS-Sp.arg3+/pUC19*, respectively. Then, $159 \mathrm{bp}$ of the Saccharomyces cerevisiae ADH1 terminator was amplified from pAW8_TAP [27] using primers P5 and P6 and the fragment cloned into SalI/SpeI restricted $P_{a d h 1}$-MCSSp.arg3+/pUC19*, $P_{\text {adh } 15}$-MCS-Sp.arg3+/pUC19* and $P_{\text {adh } 81}$-MCS-Sp.arg3+/pUC19* to create $P_{\text {adh } 1}-\mathrm{MCS}-T_{A D H 1}-$ Sp.arg $3+/$ pUC19*,$P_{\text {adh } 15}-\mathrm{MCS}-\mathrm{T}_{A D H 1}-$ Sp.arg $3+/ \mathrm{pUC19*}$ and $P_{\text {adh } 81^{-}}$ MCS-T ${ }_{A D H 1}-$ Sp.arg3+/pUC19*, respectively (MCS = NdeI-XmaI-SacI-PstI-SalI) (Figure S1).

\subsection{AID-Tagging Constructs}

The IAA17 degron tag sequence was amplified from the plasmid template pMK43 [1] using primers P7 and P8 and the fragment cloned into SphI/AscI restricted pAW8_TAP [27], replacing the TAP tag sequence, to create pAW8-aid. Primer P7 encodes a ten amino acid flexible linker sequence (Gly-Gly-Ser-Gly-Gly-Ser-Gly-Ser-Gly-Ala). Primer P8 replaces restriction enzyme site AscI with NheI. To help identify the presence of the kanMX6 antibiotic selection marker, the plasmid was renamed pAW8-aid:kanMX6. 533 bp of the S. pombe urg1 terminator sequence was amplified from total $S$. pombe genomic DNA using primers P9 and P10 and the product cloned into the NheI/BglII restricted pAW8-aid:kanMX6, replacing the $S$. cerevisiae ADH1 terminator sequence, to create $p A W 8$-aid-Turg1:kanMX6. Primer P10 inserted the restriction enzyme recognition site XmaI adjacent to the NheI site. The $157 \mathrm{bp}$ DSR element of the S. pombe spo5 gene as identified by Harigaya et al. [20] was amplified from pAW8ENdeI-spo5DSR [23] using primers P11 and P12 the product cloned into NheI/XmaI restricted pAW8-aid-Turg1:kanMX6 to create $p A W 8$-aid-spo5DSR-Turg1:kanMX6. Three copies of the human influenza hemagglutinin (HA) protein tag sequence were amplified from plasmid pAW8_3HA [28] using primers P13 and P14 or P13 and P15 the products cloned into NheI-restricted $p A W 8$-aid-Turg1:kanMX6 and NheI-restricted pAW8-aid-spo5DSRTurg1:kanMX6, respectively, to create $p A W 8$-aid-3HA-Turg1:kanMX6 and pAW8-aid-3HAspo5DSR-Turg1:kanMX6. The forward primer P13 removed the AID-tag stop codon and inserted a small flexible six amino acid linker (Gly-Ala-Gly-Ala-Gly-Ala) between the AID and 3HA tags. Five copies of the human c-myc (Мyc) epitope were amplified from plasmid pAW8_13Myc [27] using primers P16 and P17 or P16 and P18 and the resulting fragments cloned into NheI-restricted pAW8-aid-Turg1:kanMX6 and NheI-restricted pAW8-aid-spo5DSRTurg1:kanMX6, respectively, to create $p A W 8$-aid-5Myc-Turg1:kanMX6 and $p A W 8$-aid-5Mycspo5DSR-Turg1:kanMX6. The forward primer P16 removed the AID-tag stop codon and inserted a small flexible six amino acid linker (Gly-Ala-Gly-Ala-Gly-Ala) between the AID and 5Myc tags. A sequence encoding a single copy of the simian virus 5-derived V5 tag was human codon optimised and gene synthesised and cloned into NheI-restricted pAW8-aid-Turg1:kanMX6 to create pAW8-aid-V5-Turg1:kanMX6. A second sequence was synthesised allowing cloning into NheI-restricted pAW8-aid-spo5DSR-Turg1:kanMX6 to create $p A W 8$-aid-V5-spo5DSR-Turg1:kanMX6. The synthesised sequences removed the AID-tag stop codon and inserted a small flexible six amino acid linker (Gly-Ala-Gly-Ala-Gly-Ala) between the AID and V5 tags.

A mutated version of spo5DSR element was designed to contain two additional DSR core motifs and was termed 'eDSR' (Figure S2). A series of eDSR fragments compatible for cloning into plasmids $p A W 8$-aid-Turg1:kanMX6, pAW8-aid-3HA-Turg1:kanMX6, pAW8aid-5Myc-Turg1:kanMX6 and pAW8-aid-V5-Turg1:kanMX6 were synthesised and cloned into their respective NheI-restricted plasmids to create $p A W 8$-aid-eDSR-Turg1:kanMX6, pAW8-aid-3HA-eDSR-Turg1:kanMX6, pAW8-aid-5Myc-eDSR-Turg1:kanMX6 and pAW8-aidV5-eDSR-Turg1:kanMX6.

All plasmid constructs were confirmed by sequencing. All plasmid constructs and sequences are available from Addgene (see Figure 1E for Addgene ID numbers). 


\subsection{Yeast Transformations}

All yeast transformations were performed using a lithium acetate method [28]. For insertion of the phleomycin/bleomycin resistance cassette adjacent to the arg3-D4 locus, a linear PCR fragment generated using primers P19 and P20 and the plasmid template pFA6a-bleMX6 [29] was used to transform strain arg3-D4 (AW1502). After selection on YEA plates supplemented with $30 \mathrm{mg} / \mathrm{mL}$ phleomycin, transformants were confirmed by genomic PCR and sequencing. This created strain AW1655 (bleMX6-arg3-D4) (Figure S1). To fuse the AID-tag at the C-terminus of Mcm4 and Rad52, primers P21 and P22 (Mcm4) or primers P23 and P24 (Rad52) were used to generate linear PCR fragments from the relevant pAW8-aid-Turg1:kanMX6 plasmid described above and used to transform strain AW279 $(\mathrm{WT} \mathrm{h}+)$. Transformants were selected on YEA plates supplemented with $200 \mathrm{mg} / \mathrm{mL}$ G418 and confirmed using genomic PCR and sequencing.

\subsection{Spot Tests}

Cell cultures grown in YE media $30^{\circ} \mathrm{C}$ in YE medium were adjusted to a density of $1 \times 10^{7}$ cells $/ \mathrm{mL}$. A series of 10 -fold dilutions in sterile water was performed for each strain and $7 \mu \mathrm{L}$ drops were spotted onto YEA medium plates with or without supplements.

\subsection{Preparation of Total Cell Extract and Western Blot Analysis}

Preparation of cell extracts for SDS-PAGE and Western blotting was performed as previously described [30] except $\mathrm{NaN}_{3}$ was added to the cell culture sample to a final concentration of $0.1 \%$ prior to centrifugation. For protein detection, the following commercially available antibodies were used. Primary antibodies: anti-V5 (Bio-Rad, \# MCA1360) (diluted 1:5,000), anti-OsTIR1 (MBL, \#PD048) (diluted 1:1,000) and anti- $\alpha$ tubulin (Sigma, \#T5168) (diluted 1:10,000). Secondary antibodies: anti-mouse IgG HRP (Dako, \#P0260) and anti-rabbit IgG HRP (Dako, \#P0217), both diluted 1:5,000. All antibodies were incubated in phosphate-buffered saline, $0.1 \%$ v/v Tween 20 detergent (PBST) containing 3\% skimmed milk powder except anti-OsTIR1, which was incubated in PBST containing 5\% skimmed milk powder. For quantification of protein levels, Amersham ImageQuant LAS4000 Western blot imaging system was used and resulting images analysed using ImageQuant TL software (version 1.2) ( GE Healthcare, Chicago, IL, USA).

\subsection{Synthesis of 2-[5-(adamantan-1-yl)-1H-indol-3-yl]Acetic Acid (5'-adamantyl-IAA)}

$5^{\prime}$ Adamantyl-IAA was synthesised essentially as described previously [18]. Solvents were used as received, including deuterated solvents for NMR spectroscopy. NMR spectra were recorded on a Varian NMR $400\left({ }^{1} \mathrm{H} 399.5 \mathrm{MHz} ;{ }^{13} \mathrm{C}\left\{{ }^{1} \mathrm{H}\right\} 100.5 \mathrm{MHz}\right.$ or $500\left({ }^{1} \mathrm{H}\right.$ $\left.499.9 \mathrm{MHz} ; 13 \mathrm{C}\left\{{ }^{1} \mathrm{H}\right\} 125.7 \mathrm{MHz}\right)$. Chemical shifts are reported in ppm. Spectra are referenced to the corresponding protic solvent $\left({ }^{1} \mathrm{H}\right)$ or signals of the solvent $\left({ }^{13} \mathrm{C}\right)$. Reactions were monitored by TLC on commercially available glass silica gel plates (60 ̊, F254). The mobile phase was usually a solvent mixture and the visualisation was undertaken using UV light. Chromatographic purifications were carried out on an ISCO Combi Flash RF 75 or a 150 PSI purification unit. Gel columns. LC-MS purity analyses were undertaken using a $5 \mu \mathrm{m} \mathrm{C18} 110 \AA$ A column. Percentage purities were performed using a $30 \mathrm{~min}$ method in water/acetonitrile with $0.1 \%$ formic acid ( $5 \mathrm{~min}$ at $5 \%, 5-95 \%$ over $20 \mathrm{~min}, 5 \mathrm{~min}$ at $95 \%$ ) with the UV set to $254 \mathrm{~nm}$. High-resolution mass spectrometry was carried out at the University of Sussex.

2.8.1. N-(4-(adamantan-1-yl)phenyl)acetamide

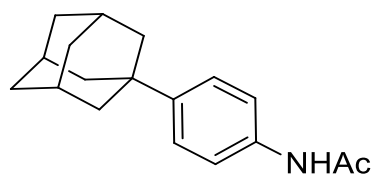


To a mixture of acetanilide $(5.07 \mathrm{~g}, 37.50 \mathrm{mmol}), 1$-bromoadamantane $(8.87 \mathrm{~g}, 41.25 \mathrm{mmol})$ and 1,1,2,2-tetrachloroethane $(50 \mathrm{~mL})$ was added zinc chloride $(10.22 \mathrm{~g}, 75.00 \mathrm{mmol})$ and the resulting mixture heated to $80^{\circ} \mathrm{C}$ and stirred for $72 \mathrm{~h}$ under an argon atmosphere. The reaction was cooled to ambient temperature and to the mixture was added $1 \mathrm{M}$ aqueous $\mathrm{HCl}(150 \mathrm{~mL})$ and dichloromethane (DCM) $(100 \mathrm{~mL})$. The resulting biphasic mixture was separated, and the aqueous phase extracted with DCM $(2 \times 100 \mathrm{~mL})$. The combined organic extracts were dried over anhydrous $\mathrm{MgSO}_{4}$, filtered, and concentrated under reduced pressure. The resulting residue was purified by automated column chromatography $(n-$ hexane/EtOAc, 100:0-30:70, $\left.100 \mathrm{~g} \mathrm{SiO}_{2}\right)$. The appropriate fractions were combined and concentrated under reduced pressure to give $N$-(4-(adamantan-1-yl)phenyl)acetamide as a white solid (5.14 g, 51\%). LCMS (UV, ESI) $R_{t}=23.52 \mathrm{~min},[\mathrm{M}-\mathrm{H}]^{+} \mathrm{m} / \mathrm{z}=270.1,88 \%$ purity. ${ }^{1} \mathrm{H}$ NMR (600 MHz, $d_{6}$-DMSO): $\delta=9.82(1 \mathrm{H}, \mathrm{s}), 7.50-7.46(2 \mathrm{H}, \mathrm{m}), 7.27-7.23(2 \mathrm{H}$, m), 2.06-2.02 (3H, m), $2.01(3 \mathrm{H}, \mathrm{s}), 1.84-1.80(6 \mathrm{H}, \mathrm{m}), 1.76-1.68(6 \mathrm{H}, \mathrm{m})$.

2.8.2. N-(4-(adamantan-1-yl)-2-iodophenyl)acetamide<smiles>CC(=O)Nc1ccc(C23CC4CC(CC(C4)C2)C3)cc1I</smiles>

To a mixture of $\mathrm{N}$-(4-(adamantan-1-yl)phenyl)acetamide $(1.82 \mathrm{~g}, 6.76 \mathrm{mmol})$, TsOH. $\mathrm{H}_{2} \mathrm{O}$ (128 mg, $0.66 \mathrm{mmol})$, and MeCN (100 mL) was added NIS (1.67 g, $7.43 \mathrm{mmol})$ and the resulting mixture was stirred under an argon atmosphere for $24 \mathrm{~h}$ at ambient temperature. The mixture was concentrated under reduced pressure and to the residue EtOAc $(50 \mathrm{~mL})$ was added and saturated aqueous $\mathrm{NaHCO}_{3}(50 \mathrm{~mL})$. The resulting biphasic mixture was separated, and the aqueous phase extracted with EtOAc $(3 \times 50 \mathrm{~mL})$. The combined organic extracts were washed with brine, dried over anhydrous $\mathrm{MgSO}_{4}$, filtered, and concentrated under reduced pressure to give a brown solid $(2.7 \mathrm{~g})$. The solid was purified by automated flash column chromatography ( $n$-hexane/EtOAc, 100:0-50:50, $30 \mathrm{~g} \mathrm{SiO}_{2}$ ). The appropriate fractions were combined and concentrated under reduced pressure to give $N$-(4-(adamantan-1-yl)-2-iodophenyl)acetamide as a light orange solid (2.62 g, 98\%). LCMS (UV, ESI) $R_{t}=25.59 \mathrm{~min},[\mathrm{M}-\mathrm{H}]^{+} \mathrm{m} / z=396.0,93 \%$ purity. ${ }^{1} \mathrm{H}$ NMR $\left(600 \mathrm{MHz}, d_{6}-\mathrm{DMSO}\right)$ : $\delta=9.36(1 \mathrm{H}, \mathrm{s}), 7.75(1 \mathrm{H}, \mathrm{d}, J=2.2 \mathrm{~Hz}), 7.36(1 \mathrm{H}, \mathrm{dd}, J=8.4,2.2 \mathrm{~Hz}), 7.30(1 \mathrm{H}, \mathrm{d}, J=8.4 \mathrm{~Hz})$, 2.07-2.02 (3H, m), $2.03(3 \mathrm{H}, \mathrm{s}), 1.85-1.82(6 \mathrm{H}, \mathrm{m}), 1.76-1.69(6 \mathrm{H}, \mathrm{m})$.

2.8.3. N-(4-(adamantan-1-yl)-2-((trimethylsilyl)ethynyl)phenyl)acetamide<smiles>CC(C)(C)C#Cc1cc(C23CC4CC(CC(C4)C2)C3)ccc1N</smiles>

To N-(4-(adamantan-1-yl)-2-iodophenyl)acetamide (1.00 g, $2.53 \mathrm{mmol}), \mathrm{Pd}\left(\mathrm{PPh}_{3}\right)_{2} \mathrm{Cl}_{2}$ (44 mg, $0.063 \mathrm{mmol})$, and CuI (24 mg, $0.127 \mathrm{mmol})$ was added THF:NEt 3 (1:1) (25 mL) followed by trimethylsilyacetylene $(456 \mu \mathrm{L}, 3.29 \mathrm{mmol})$. The resulting mixture was stirred under argon for $16 \mathrm{~h}$ at ambient temperature. The reaction was concentrated under reduced pressure and purified by automated flash column chromatography (n-hexane/EtOAc, 100:0$\left.60: 40,40 \mathrm{~g} \mathrm{SiO}_{2}\right)$. The appropriate fractions were combined and concentrated under reduced pressure to give $N$-(4-(adamantan-1-yl)-2-((trimethylsilyl)ethynyl)phenyl)acetamide as a brown solid (740 $\mathrm{mg}, 80 \%$ ). LCMS (UV, ESI) $R_{t}=29.64 \mathrm{~min},[\mathrm{M}-\mathrm{H}]^{+} \mathrm{m} / z=$ did not ionise, 94\% purity. ${ }^{1} \mathrm{H}$ NMR (600 MHz, $d_{6}$-DMSO): $\delta=9.13(1 \mathrm{H}, \mathrm{s}), 7.60(1 \mathrm{H}, \mathrm{d}, J=8.8 \mathrm{~Hz})$, 7.39-7.34 (2H, m), 2.08-2.03 (6H, m), 1.85-1.81 (6H, m), 1.74-1.70 (6H, m), $0.25(9 \mathrm{H}, \mathrm{s})$. 
2.8.4. 5-(Adamantan-1-yl)-1H-indole

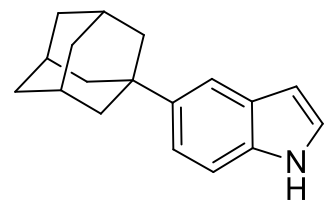

To a mixture of $\mathrm{N}$-(4-(adamantan-1-yl)-2-((trimethylsilyl)ethynyl)phenyl)acetamide $(630 \mathrm{mg}, 1.72 \mathrm{mmol})$ and anhydrous THF $(16 \mathrm{~mL})$ was added tetrabutylammonium fluoride (TBAF) (1 M in THF) $(2.50 \mathrm{~mL})$. The resulting mixture was heated to $80^{\circ} \mathrm{C}$ and stirred for $16 \mathrm{~h}$ under an argon atmosphere. The reaction was cooled to ambient temperature and to the mixture was added EtOAc $(15 \mathrm{~mL})$ and water $(15 \mathrm{~mL})$. The resulting biphasic mixture was separated, and the aqueous phase extracted with EtOAc $(3 \times 15 \mathrm{~mL})$. The combined organic extracts were washed with brine $(25 \mathrm{~mL})$, dried over anhydrous $\mathrm{MgSO}_{4}$, filtered, and concentrated under reduced pressure to give a brown oil $(500 \mathrm{mg})$. The resulting residue was purified by automated flash column chromatography ( $n$-hexane/EtOAc, 100:0$70: 30,24 \mathrm{~g} \mathrm{SiO}_{2}$ ). The appropriate fractions were combined and concentrated under reduced pressure to give 5-(adamantan-1-yl)- $1 \mathrm{H}$-indole as a pale yellow solid (330 mg, 76\%). LCMS (UV, ESI) $R_{t}=27.19 \mathrm{~min},[\mathrm{M}-\mathrm{H}]^{+} \mathrm{m} / z=252.1,91 \%$ purity. ${ }^{1} \mathrm{H}$ NMR $\left(600 \mathrm{MHz}, d_{6}\right.$-DMSO): $\delta=10.88(1 \mathrm{H}, \mathrm{s}), 7.44(1 \mathrm{H}, \mathrm{s}), 7.30(1 \mathrm{H}, \mathrm{d}, J=8.8 \mathrm{~Hz}), 7.26(1 \mathrm{H}, \mathrm{m}), 7.14(1 \mathrm{H}, \mathrm{dd}, J=8.8$, $1.8 \mathrm{~Hz}), 6.35(1 \mathrm{H}, \mathrm{m}), 2.09-2.05(3 \mathrm{H}, \mathrm{m}), 1.92-1.89(6 \mathrm{H}, \mathrm{m}), 1.78-1.72(6 \mathrm{H}, \mathrm{m})$.

2.8.5. Methyl 2-(5-(adamantan-1-yl)-1H-indol-3-yl)-2-oxoacetate<smiles>CC(=O)C(=O)c1c[nH]c2ccc(C34CC5CC(CC(C5)C3)C4)cc12</smiles>

To a mixture of 5-(adamantan-1-yl)- $1 H$-indole $(294 \mathrm{mg}, 1.17 \mathrm{mmol})$ and anhydrous $\mathrm{Et}_{2} \mathrm{O}(10 \mathrm{~mL})$ was added oxalyl chloride $(148 \mu \mathrm{L}, 1.75 \mathrm{mmol})$ and the resulting mixture stirred for $16 \mathrm{~h}$ at ambient temperature under argon. To the mixture was added $\mathrm{MeOH}$ $(10 \mathrm{~mL})$ and the resulting mixture stirred for $15 \mathrm{~min}$. The resulting mixture was concentrated under reduced pressure to give a yellow solid of sufficient purity to be utilised in the subsequent reaction without further manipulation.

2.8.6. 2-[5-(Adamantan-1-yl)-1H-indol-3-yl]Acetic Acid<smiles>O=C(O)Cc1c[nH]c2ccc(C34CC5CC(CC(C5)C3)C4)cc12</smiles>

To a mixture of crude methyl 2-(5-(adamantan-1-yl)- $1 H$-indol-3-yl)-2-oxoacetate (395 mg, $1.17 \mathrm{mmol}), \mathrm{NaH}_{2} \mathrm{PO}_{2} \cdot \mathrm{H}_{2} \mathrm{O}$ (1.24 g, $\left.11.70 \mathrm{mmol}\right)$, and $\mathrm{H}_{2} \mathrm{O} / 1$,4-dioxane (1:5) $(10 \mathrm{~mL})$ was added $\mathrm{Pd} / \mathrm{C}(10 \%)(128 \mathrm{mg}, 0.12 \mathrm{mmol})$. The resulting mixture was heated to $100{ }^{\circ} \mathrm{C}$ and stirred for $72 \mathrm{~h}$ under an argon atmosphere. The reaction was cooled to ambient temperature and filtered through Celite ${ }^{\circledR}$, washing with EtOAc $(25 \mathrm{~mL})$. To the mixture was added $2 \mathrm{M}$ aqueous $\mathrm{HCl}(25 \mathrm{~mL})$ and the resulting biphasic mixture separated. The organic phase was washed with brine $(25 \mathrm{~mL})$, dried over anhydrous $\mathrm{MgSO}_{4}$, filtered, and concentrated under reduced pressure to give a light red gum $(550 \mathrm{mg})$. The resulting residue was purified by automated flash column chromatography ( $n$-hexane/EtOAc, 100:0-70:30, $24 \mathrm{~g} \mathrm{SiO}_{2}$ ). The appropriate fractions were combined and concentrated under reduced pressure to give 2-(5-(adamantan-1-yl)- $1 H$-indol-3-yl)acetic acid as a white solid (129 mg, 36\% over 2 steps). LCMS (UV, ESI) $R_{t}=22.49 \mathrm{~min},[\mathrm{M}-\mathrm{H}]^{+} \mathrm{m} / \mathrm{z}=310.0,96 \%$ purity. ${ }^{1} \mathrm{H}$ NMR $\left(600 \mathrm{MHz}, d_{6}-\mathrm{DMSO}\right): \delta=12.11(1 \mathrm{H}, \mathrm{s}), 10.73(1 \mathrm{H}, \mathrm{s}), 7.42(1 \mathrm{H}, \mathrm{m}), 7.27(1 \mathrm{H}, \mathrm{d}$, 
$J=8.8 \mathrm{~Hz}), 7.18-7.13(2 \mathrm{H}, \mathrm{m}), 3.62(2 \mathrm{H}, \mathrm{s}), 2.10-2.05(3 \mathrm{H}, \mathrm{m}), 1.93-1.89(6 \mathrm{H}, \mathrm{m}), 1.78-1.72$ $(6 \mathrm{H}, \mathrm{m}) .{ }^{13} \mathrm{C}$ NMR $\left(151 \mathrm{MHz}, d_{6}-\mathrm{DMSO}\right): \delta=173.2,141.1,134.3,126.9,123.8,118.5,113.7$, $110.8,107.7,43.4,36.4,35.5,31.0,28.5$. HRMS (ESI-[+Na]) $m / z$ : Calcd for $\mathrm{C}_{20} \mathrm{H}_{23} \mathrm{NNaO}_{2}$ 332.1626; Found 332.1615.

\section{Results}

\subsection{Integration of OsTIR $1^{W T}$ and OsTIR $1^{F 74 A}$ Expression Constructs at the arg3-D4 Locus}

OsTIR1 is the most commonly used AID system auxin receptor F-box protein. Moreover, in budding yeast, OsTIR1 has been shown to work at high temperatures and expression of OsTIR1 from the constitutive alcohol dehydrogenase gene $(A D H 1)$ promoter does not affect cell growth [1]. We therefore wished to test OsTIR1 not fused to Skp1 in a new fission yeast AID system.

We developed a set of plasmids to integrate the OsTIR $1^{\mathrm{WT}}$ and OsTIR ${ }^{\mathrm{F}}{ }^{\mathrm{F}} \mathrm{A}$ expression constructs at the S. pombe arg3-D4 locus. We sequenced the arg3-D4 locus [24] (Figure S1) to determine the region deleted and synthesised this sequence and approximately 500bp flanking the deletion site. These flanking sequences allow integration of the deleted arg3 gene fragment at the arg3-D4 locus and transformants can be screened for arginine prototrophy. Synthesis of this fragment allowed for insertion of an MCS (NheI-NdeI-SphIXmaI-SacI-PstI-SalI-SpeI) and the removal of restriction enzyme recognition sites from the $\arg 3$ gene sequence which may hinder subsequent cloning events. The synthesised gene fragment was cloned into pUC19* (where * denotes the removal of the NdeI restriction enzyme recognition site from the plasmid backbone) to create MCS-Sp.arg3+/pUC19*.

Expression cassettes consisting of an MCS site flanked by approximately $820 \mathrm{bp}$ of the $S$. pombe adh1 promoter and $160 \mathrm{bp}$ of the $S$. cerevisiae ADH1 terminator sequences were constructed. Three individual constructs were created each containing different adh1 promoter strengths. The strongest promoter strength was the wild-type adh1 promoter sequence $\left(P_{a d h 1}\right)$ followed by adh15 promoter $\left(P_{a d h 15}\right)$, a weak derivative of the $a d h 1$ promoter [11] and finally the $a d h 81$ promoter $\left(P_{a d h 81}\right)$ a much weaker derivative of $P_{a d h 1}$ [31]. These three expression constructs were cloned into MCS-Sp.arg3+/pUC19* to create $\mathrm{P}_{\mathrm{adh} 1}-\mathrm{MCS}-\mathrm{T}_{\mathrm{ADH} 1}-$ Sp.arg3+/pUC19*, $\mathrm{P}_{\mathrm{adh} 15}-\mathrm{MCS}-\mathrm{T}_{\mathrm{ADH} 1}-$ Sp.arg3+/pUC19* and $\mathrm{P}_{\mathrm{adh} 81}-\mathrm{MCS}-\mathrm{T}_{\mathrm{ADH1}}-$ Sp.arg3+/pUC19*, respectively (MCS = NdeI-XmaI-SacI-PstI-SalI).

For the new AID system, human-codon optimised OsTIR1 ${ }^{\text {WT }}$-NLS (NLS: nuclear localisation signal) was gene synthesised and cloned into $\mathrm{P}_{\mathrm{adh} 1}-\mathrm{MCS}-\mathrm{T}_{\mathrm{ADH} 1}-\mathrm{Sp}$.arg3+/pUC19* to create $\mathrm{P}_{\mathrm{adh} 1}$-OsTIR1 ${ }^{\mathrm{WT}}$-NLS-T ${ }_{\mathrm{ADH} 1}-\mathrm{Sp} \cdot \arg 3+/ \mathrm{pUC19*}$. For AID version 2 (AID2), humancodon optimised OsTIR1 ${ }^{\text {F74A }}$-NLS was gene synthesised and cloned into $\mathrm{P}_{\text {adh1 }}$-MCS$\mathrm{T}_{\mathrm{ADH} 1}-$ Sp.arg3+/pUC19* to create $\mathrm{P}_{\mathrm{adh} 1}-\mathrm{OsTIR1}{ }^{\mathrm{F} 74 \mathrm{~A}}-\mathrm{NLS}-\mathrm{T}_{\mathrm{ADH} 1}-$ Sp.arg3+/pUC19*. The OsTIR1 ${ }^{\text {F74A }}$ mutation corresponds to the AtTIR1 auxin-binding site mutation F79A identified by Yamada et al. [18] and used by Nishimura et al. [19] (see also Figure S3). The NLS directs the system to specifically deplete nuclear proteins. The plasmids $\mathrm{P}_{\text {adh1 }}-\mathrm{OsTIR} 1^{\mathrm{WT}}$ NLS-T ${ }_{\mathrm{ADH} 1}$-arg3+/pUC19* and $\mathrm{P}_{\mathrm{adh} 1}$-OsTIR1 ${ }^{\text {F74A }}-\mathrm{NLS}-\mathrm{T}_{\mathrm{ADH} 1}-$ Sp.arg3+/pUC19* were restricted with NotI restriction enzyme and the linear fragments used to transform AW1655 (bleMX6-arg3-D4). Transformants were screened for arginine prototrophy and successful integrants confirmed by genomic PCR and sequencing (Figure 1B). This created strains Padh1-OsTIR1 $^{\text {WT }}$ (AW1762) and Padh1-OsTIR1 ${ }^{\text {F74A }}$ (AW1703). Expression of OsTIR1 ${ }^{\text {WT }}$ and OsTIR1 ${ }^{\text {F74A }}$ was confirmed by immunoblot analysis and, as shown previously [17], the F74A substitution does not significantly affect stability of the TIR1 protein (Figure 1C). Expression of OsTIR $1^{\mathrm{WT}}$ or OsTIR $1^{\mathrm{F} 74 \mathrm{~A}}$ from the strong constitutive $a d h 1$ promoter does not affect cell growth rate, with doubling times of Padh1-OsTIR $1^{\text {WT }}$ and Padh1-OsTIR1 ${ }^{\text {F74A }}$ cells comparable to wild-type cells (Figure 1D). As cellular levels of TIR1 have been shown to be a limiting factor for auxin response [10,32,33], we predict that increasing the TIR1 expression level will increase AID efficiency. 


\subsection{Plasmids for C-Terminal AID Tagging}

To allow the C-terminal tagging of proteins of interest, we developed a set of plasmids containing the full-length $A$. thaliana auxin-responsive AUX/IAA protein IAA17 gene sequence. The tagging constructs also include a sequence encoding a flexible 10-amino-acid linker (Gly-Gly-Ser-Gly-Gly-Ser-Gly-Ser-Gly-Ala) that will be inserted between the protein of interest and the AID-tag. This was included to help minimise loss of either target protein or AID-tag function. The plasmids also included approximately $530 \mathrm{bp}$ of $S$. pombe urg 1 downstream sequence to act as a transcription terminator and the antibiotic resistance cassette, kanMX6, which allows selection of G418-resistant cells (Figure 1E).

To detect AID-tagged proteins by immunoblotting using commercial antibodies, a series of additional short epitope tag sequences were fused to the C-terminus of the AID tag. These tags included three copies of the influenza virus hemagglutinin (HA) epitope tag (3HA), five copies of the human c-myc (Myc) epitope (5Myc) and a single copy of the $\mathrm{V} 5$ tag derived from the $\mathrm{P}$ and $\mathrm{V}$ protein of the simian virus 5 (SV5, a paramyxovirus). The AID and epitope tags were separated by a short 6-amino-acid flexible linker (Gly-Ala-GlyAla-Gly-Ala) (Figure 1E).

To provide an option to constitutively reduce AID-tagged protein expression levels from the constructs outlined above, we inserted the $157 \mathrm{bp}$ DSR element derived from the S. pombe spo5 gene directly downstream of the tag stop codon (Figure 1E). Levels of yEGFP expressed from the uracil-inducible $u$ rg 1 locus are reduced approximately 25 -fold by the spo5DSR element compared to the no DSR control [23]. The spo5DSR element contains 5 copies of the hexanucleotide motif U(U/C)AAAC shown to be highly enriched in DSR elements [22]. To increase the efficiency of the spo5DSR element and further reduce AID-tagged protein levels, two sequences similar to the core motif were mutated to match the hexanucleotide DSR motif (Figure S2). This new DSR element, termed 'eDSR', was introduced into the AID-plasmids, replacing spo5DSR. The full set of plasmids are shown in Figure 1E.

The AID-tagging constructs were cloned into the Cre-expression plasmid pAW8, allowing for rapid and efficient C-terminal fusion of the AID-tag using Cre-mediated cassette exchange [27]. These tagging construct plasmids are also compatible with PCRbased genomic strategies with the primer sequences required to amplify the series of AID constructs shown in Figure 1F.

\subsection{Testing the New S. pombe AID/OsTIR1(WT) System}

As a target of degradation, we used Mcm4, a component of the DNA replication MCM complex to test the new AID/OsTIR1 system. Mcm4 is an essential protein and $m c m 4 \Delta$ cells are inviable. The $i$-AID system (Padh15-Skp1-AtTIR1) was used previously to deplete Mcm4 [10] and allows direct comparison of the efficiency of the two systems. Mcm4 was successfully C-terminally AID-tagged using a PCR-mediated approach using the plasmids outlined in Figure 1E. The mcm4-AID strain was then crossed to strains expressing Padh15skp1-AtTIR1 or Padh1-OsTIR1 ${ }^{\text {WT }}$ and the resulting strains were spotted onto a YEA plate or YEA plates containing the synthetic auxin NAA at $500 \mu \mathrm{M}$ and the plates were incubated at $25^{\circ} \mathrm{C}, 30^{\circ} \mathrm{C}$ or $36^{\circ} \mathrm{C}$ for 3 days (Figure 1G). Unlike Skp1-AtTIR1 expressing i-AID cells, expression of OsTIR1 from the strong adh1 constitutive promoter showed no inhibition in cell growth, as has previously been observed for budding yeast [1]. Although mcm4AID skp1-AtTIR1 cell growth was severely inhibited at $25^{\circ} \mathrm{C}$ by the presence of auxin, increasing the temperature to $30^{\circ} \mathrm{C}$ reduced this inhibition and growth rates were more similar to WT (Figure 1G). However, mcm4-aid OsTIR1 ${ }^{\text {WT }}$ cells showed complete loss of cell viability when grown in the presence of auxin at $25^{\circ} \mathrm{C}$ and, more importantly, also at $30^{\circ} \mathrm{C}$ (Figure 1G). The relative thermostability of OsTIR1 compared to AtTIR1 has been previously reported [1] and explains the improved performance observed for our new AID system with respect to temperature. Overall, these data clearly demonstrate the significant improvement in degradation efficiency when using Padh1-OsTIR1 as opposed to systems involving Padh15-skp1-AtTIR1. 


\subsection{Further Characterisation of the S. pombe AID/OsTIR $1^{\text {WT }}$ System}

To determine the rate and percentage reduction in AID-tagged protein levels following addition of auxin, we created the strains $m c m 4-A I D-5 m y c$ and $m c m 4-A I D-V 5$ using the plasmids outlined in Figure 1E (we were unable to create mcm4-AID-3HA). As we found the signal-to-noise ratio of the anti-V5 antibody was significantly higher than that for anti-Myc, $m c m 4-A I D-V 5$ cells were used to study protein levels. Complete inviability in the presence of NAA of $m c m 4-A I D-V 5$ OsTIR $1^{\text {WT }}$ cells demonstrate that the V5 epitope tag does not compromise AID degron function (Figure 2A).

mcm4-AID-V5 OsTIR $1^{\text {WT }}$ cells were grown to mid-log phase, synthetic auxin NAA was added to a final concentration of $500 \mu \mathrm{M}$ and total protein extracted at various timepoints. Total protein was also extracted from $m c m 4^{+}$(untagged Mcm4) and mcm4-AID-V5 cells with no OsTIR1. Following immunoblotting using anti-V5 antibodies and, as a loading control, anti-tubulin, the degradation kinetics were determined (Figure 2B). Quantification of AID-tagged protein levels reveal a 14\% reduction in Mcm4-AID-V5 protein levels in cells exogenously expressing OsTIR1 in the absence of NAA. The chronic auxin-independent depletion of AID-tagged proteins by TIR1 has been extensively reported [34-36]. After addition of NAA a rapid depletion of cellular levels of Mcm4-AID-V5 was observed with a 90\% reduction after $15 \mathrm{~min}$. However, detectable levels of Mcm4-AID-V5 were observed $3 \mathrm{~h}$ after addition of NAA with approximately $5 \%$ of protein remaining (Figure 2C).

Using Mcm4 to assess the efficiency of the OsTIR1 auxin-inducible degron system is limited because $\mathrm{Mcm} 4$ is an essential protein and a reduction in protein level by approximately $95 \%$ leads to complete cell inviability (Figure 2A,C). Therefore, we chose to also study the DNA repair protein Rad52 because, unlike Mcm4, Rad52 is a non-essential protein and rad52 $\Delta$ cells are viable. Depletion of Rad52 can be monitored phenotypically as rad52 $\Delta$ cells display a slow-growth phenotype and are hyper sensitive to DNA damaging agents such as hydroxyurea (HU) [37].

We successfully AID-tagged Rad52 via a PCR-based approach using the plasmids outlined in Figure 1E as PCR templates. Spot test analysis shows that rad52-AID and rad52$A I D-V 5$ cells grow at WT rates and show WT levels of resistance to the genotoxic agent HU, meaning that the AID and AID-V5 tags do not adversely affect Rad52 function (Figure 2D). This is in contrast to rad52 $\Delta$ cells that are both slow growing and are hypersensitive to HU treatment with significant levels of cell death observed at low HU concentrations ( $2 \mathrm{mM})$. In the presence of NAA, however, rad52-AID OsTIR $1^{W T}$ and rad52-AID-V5 OsTIR $1^{W T}$ cells exhibit increased sensitivity to HU when compared to untagged Rad52 control cells, but only at higher HU concentrations $(4 \mathrm{mM})$. This indicates that significant levels of Rad52-AID protein remain when NAA is present (Figure 2D).

We used rad52-AID-V5 cells to monitor protein levels. rad52-AID-V5 OsTIR1 ${ }^{W T}$ cells were grown to mid-log phase, NAA was added to a final concentration of $500 \mu \mathrm{M}$ and total protein extracted at various timepoints. As with $m c m 4-A I D-V 5$ OsTIR $1^{\text {WT }}$ cells, chronic auxin-independent depletion of Rad52-AID-V5 by OsTIR1 was observed with protein levels reduced by approximately $14 \%$ compared to rad52-AID-V5 cells (Figure 2F). As with $\mathrm{Mcm} 4$, the addition of NAA induced a rapid depletion of Rad52-AID-V5, with levels reduced by $78 \%$ within 15 min but Rad52-AID-V5 protein levels were detectable even after overnight treatment (18 h). Residual levels of Rad52-AID-V5 in cells expressing OsTIR1 in the presence of NAA are comparable to those seen for Mcm4-approximately $4 \%$ relative to no TIR1 (Figure 2F). 
(A)

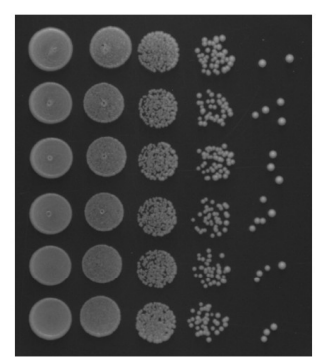

NO NAA

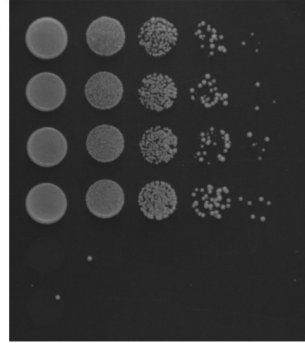

mcm4+

mcm4-AID

mcm4-AID-V5

mcm4+ mcm4-AID + OsTIR1 ${ }^{w T}$ mcm4-AID-V5

$500 \mu$ M NAA

(B)

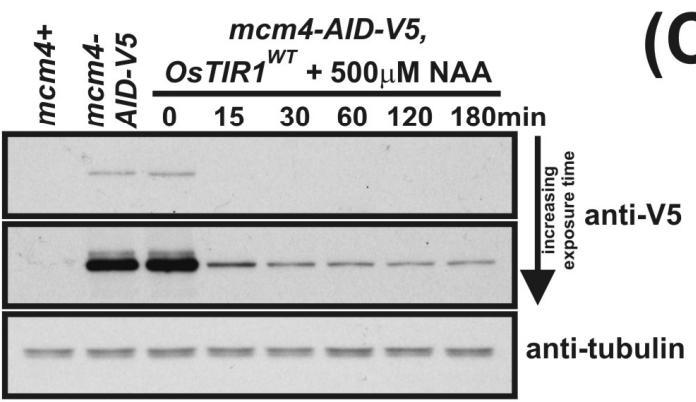

(C)

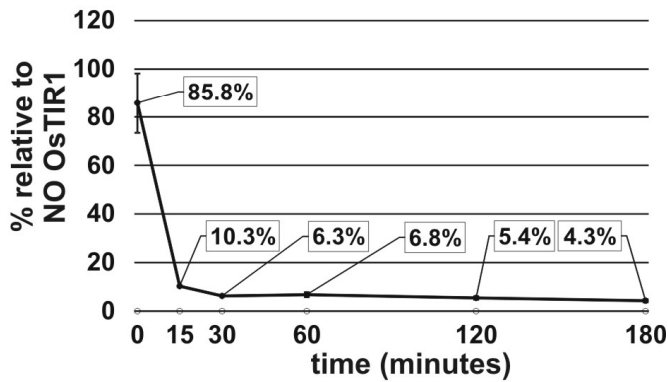

(D)
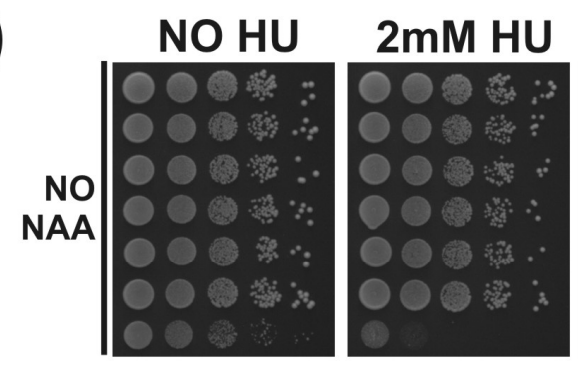

$4 \mathrm{mM} \mathrm{HU}$
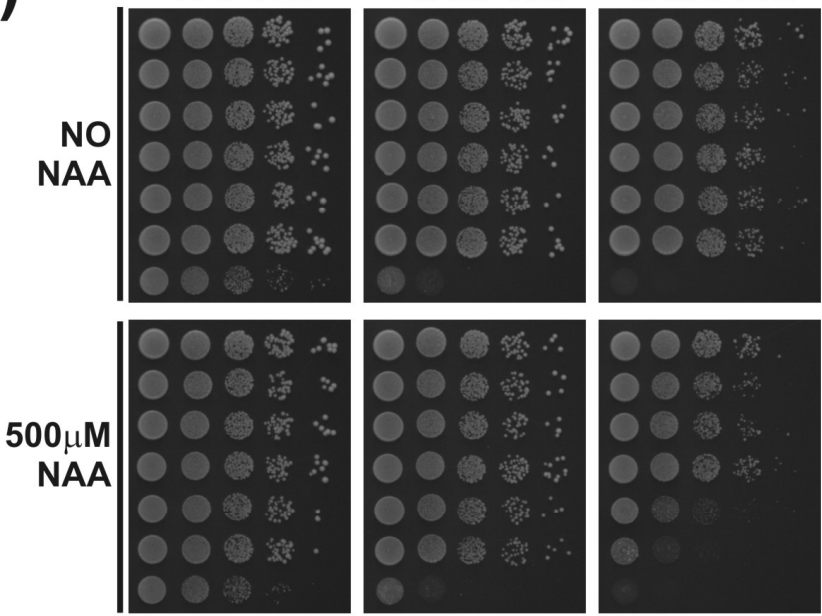

rad52+

rad52-AID

rad52-AID-V5

rad52+

rad52-AID

rad52-AID-V5

rad52-delete

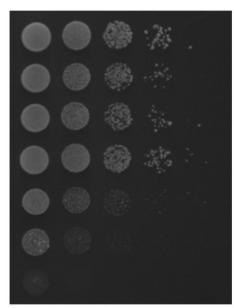

rad52+

rad52-AID

rad52-AID-V5

rad52+

rad52-AID

rad52-AID-V5

rad52-delete

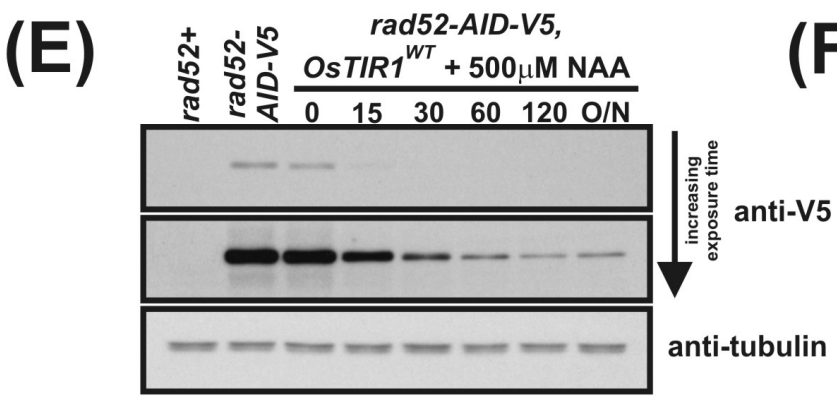

(F)

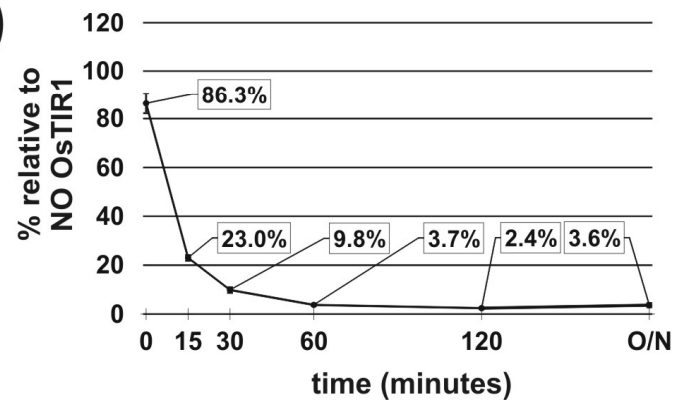

Figure 2. Regulation of Mcm4 and Rad52 activity using AID/OsTIR1 ${ }^{\mathrm{WT}}$. (A) Isogenic strains with indicated genotypes (mcm4+ (AW279), mcm4-AID (AW1893), mcm4-AID-V5 (AW1899), OsTIR1 ${ }^{\text {WT }}$ (AW1762), mcm4-AID OsTIR1 ${ }^{\text {WT }}$ (AW1923), 
mcm4-AID-V5 OsTIR1 ${ }^{\text {WT }}$ (AW1935)) were spotted onto YEA plates and plates containing $500 \mu \mathrm{M}$ NAA. Plates were grown at $30^{\circ} \mathrm{C}$ for 3 days. The experiment was repeated and similar results were obtained. (B) Representative immunoblot on total protein extract from mcm4+ (AW279), mcm4-AID-V5 (AW1899) and mcm4-AID-V5 OsTIR1 ${ }^{W T}$ (AW1935) cells treated with $500 \mu \mathrm{M}$ NAA and sampled at indicated time points and detected using anti-V5 antibody. Anti-tubulin was used as a loading control. (C) Graph of quantified anti-V5 signal normalised against anti-tubulin signal for (B) (data represented as the mean values + / - SD of three independent experiments). (D) Isogenic strains with indicated genotypes (rad52+ (AW279), rad52AID (AW1901), rad52-AID-V5 (AW1907), OsTIR1 ${ }^{W T}$ (AW1762), rad52-AID OsTIR1 ${ }^{W T}$ (AW1990), rad52-AID-V5 OsTIR1 ${ }^{\text {WT }}$ (AW1996), rad52 $\Delta$ (AW1581)) were spotted onto YEA plates and plates containing $500 \mu \mathrm{M}$ NAA and/or genotoxic agent hydroxyurea $(\mathrm{HU})$ at indicated concentrations. Plates were grown at $30{ }^{\circ} \mathrm{C}$ for 3 days. The experiment was repeated and similar results were obtained. (E) Representative immunoblot on total protein extract from rad52+ (AW279), rad52-AID-V5 (AW1907) and rad52-AID-V5 OsTIR1 ${ }^{W T}$ (AW1996) cells treated with $500 \mu \mathrm{M}$ NAA and sampled at the indicated time points and detected using anti-V5 antibody. Anti-tubulin was used as a loading control. (F) Graph of quantified anti-V5 signal normalised against anti-tubulin signal (data represented as the mean values +/ - SD of three independent experiments).

Overall, these data demonstrate the effectiveness of the AID/OsTIR1 ${ }^{\text {WT }}$ system. AIDtagged proteins are rapidly degraded in the presence of NAA with overall levels reduced approximately 20 -fold. However, detectable levels of AID-tagged protein remain. For an essential protein such as $\mathrm{Mcm} 4$, this residuary level is low enough to render the cell inviable and thus a conditional null-mutant phenotype was obtained. However, for a non-essential gene, such as Rad52, this residual level did not manifest a conditional null phenotype: NAA-treated cells only showed sensitivity to HU at concentrations that were higher than those required to kill rad52-deleted cells. Moreover, the effectiveness of the AID/OsTIR $1^{\text {WT }}$ system is potentially compromised by the NAA-independent association of AID-tagged proteins with OsTIR1, resulting in levels being reduced by approximately $14 \%$ relative to no TIR1 controls.

\subsection{Using the AID2/OsTIR1 ${ }^{\text {F74A }}$ System in S. pombe}

Recent AID systems have made use of synthetic auxin-TIR1 pairs that function orthogonally to the natural auxin signalling pathway. The systems involve an auxin-binding site mutant and a 5-substituted synthetic auxin and overcome problems associated with the original AID system, namely, the requirement for a high dose of auxin and the auxinindependent degradation of AID-tagged target proteins. The new AID systems (termed AID version 2 or 'AID2') have been characterised for use in chicken DT40 cells, budding yeast, mammalian cells and mice and have been shown to require a markedly lower ligand concentration for the association of TIR1 with the AID-tag and no detectable basal degradation of AID-tagged proteins was observed $[6,19]$. The rate of degradation has been also shown to be even quicker in AID2 compared to AID [6].

To test an AID2 in fission yeast, we construct a system analogous to that described by Yamada et al. [18]. We synthesised the 5-substituted auxin 5'adamantyl-IAA (Figure 3A) and created a strain that endogenously expresses the high-affinity-binding partner OsTIR $1^{\mathrm{F} 74 \mathrm{~A}}$, corresponding to AtTIR $1^{\mathrm{F} 9 \mathrm{~A}}$, using the arg3-D4 integration expression vector system we have developed (Figure 1B). Growth analysis showed that overexpression of OsTIR1 ${ }^{\text {F74A }}$ using the strong constitutive $a d h 1$ promoter does not affect cell growth (Figure 1D). We crossed the OsTIR $1^{F 74 A}$ strain to the mcm4-AID and mcm4-AID-V5 strains and, using spot test analysis, tested a range of concentrations of the AID2 inducer $5^{\prime}$ adamantyl-IAA $(100 \mathrm{nM}, 10 \mathrm{nM}$ and $1 \mathrm{nM})$. We found that a $5^{\prime}$ adamantyl-IAA concentration of $1 \mathrm{nM}$ was sufficient to generate a lethal phenotype and that $10 \mathrm{nM}$ was, upon microscopic examination, able to completely inhibit cell growth (Figure S5A and Figure 3B). The $10 \mathrm{nM}$ represents a 5,000-fold reduction in ligand concentration compared to OsTIR1 ${ }^{\text {WT }}$ /NAA. Interestingly, the AID2/OsTIR1 ${ }^{\mathrm{F} 74 \mathrm{~A}}$ system also functions better at the higher growth temperature of $36^{\circ} \mathrm{C}$ (Figure 3B; compare with Figure 1G). This clearly demonstrates that the AID2 OsTIR1 $1^{\mathrm{F} 4 \mathrm{~A}} / 5^{\prime}$ adamantyl-IAA system is fully functional in fission yeast and, with respect to $\mathrm{Mcm} 4$, requires a significantly lower ligand concentration compared to AID/OsTIR1 ${ }^{\text {WT }} /$ NAA. 

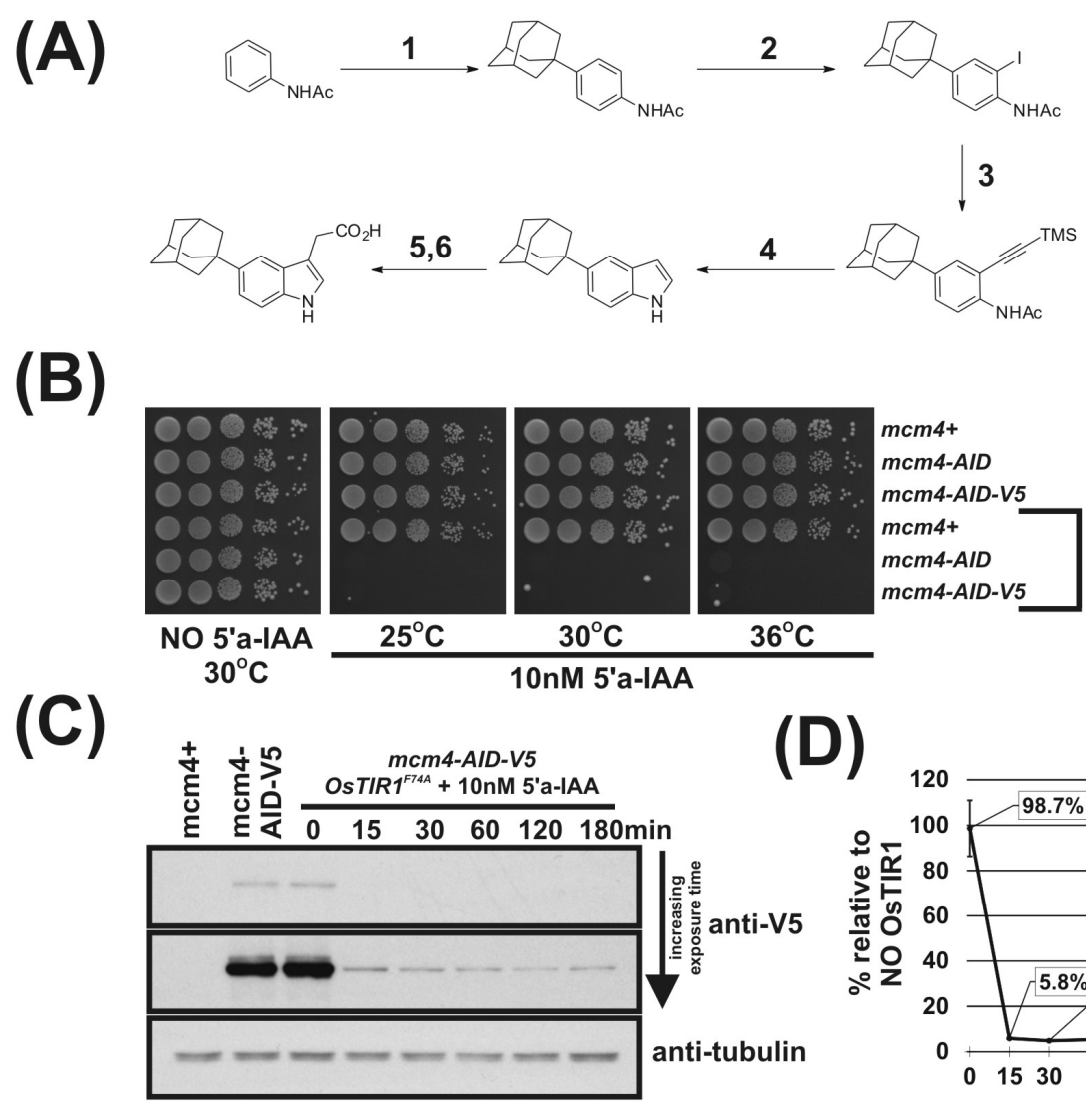

(B)

(E)

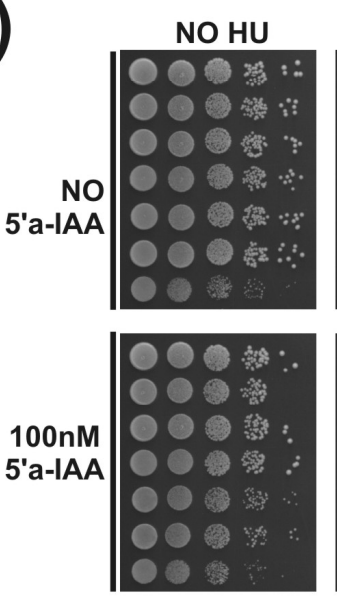

$2 \mathrm{mM} \mathrm{HU}$

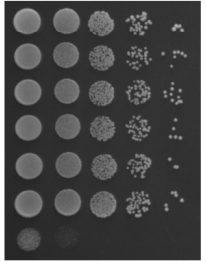

$4 \mathrm{mM} \mathrm{HU}$

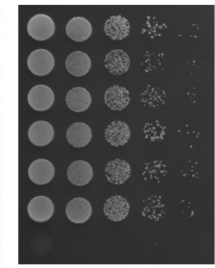

rad52+

rad52-AID

rad52-AID-V5

rad52+

rad52-AID

rad52-AID-V5

rad52-delete
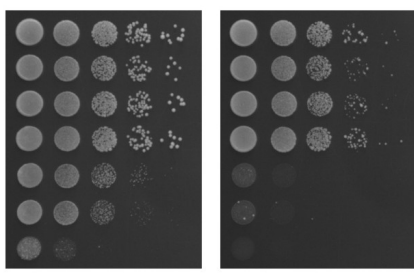

rad52+

rad52-AID

rad52-AID-V5

rad52+

rad52-AID

rad52-AID-V5

rad52-delete

(F)

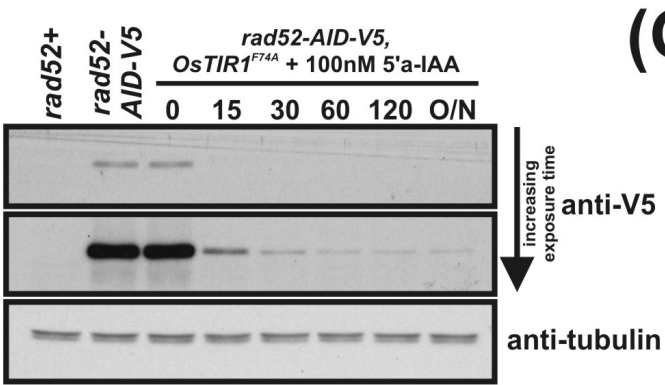

(G)

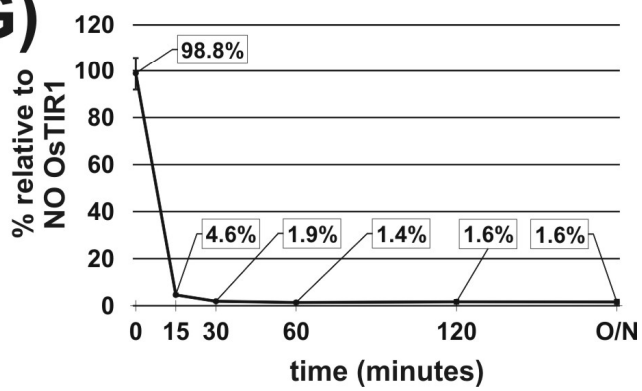

Figure 3. Regulation of Mcm4 and Rad52 activity using AID2/OsTIR1 ${ }^{\mathrm{F} 74 \mathrm{~A}}$. (A) Schematic illustration showing synthesis of 2[5-(adamantan-1-yl)-1H-indol-3-yl]acetic acid (5'adamantyl-IAA). (1) 1-Bromoadamantane, $\mathrm{ZnCl}_{2}, 1,1,2$,2-tetrachloroethane, 
$80{ }^{\circ} \mathrm{C}, 72 \mathrm{~h}, 51 \%$; (2) NIS, TsOH.H ${ }_{2} \mathrm{O}, \mathrm{MeCN}, \mathrm{rt}, 24 \mathrm{~h}, 98 \%$; (3) trimethylsilyacetylene, $\mathrm{Pd}_{\left(\mathrm{PPh}_{3}\right){ }_{2} \mathrm{Cl}_{2}, \mathrm{CuI}, \mathrm{THF}: \mathrm{NEt}}$ (1:1), $80 \%$; (4) TBAF, THF, $80{ }^{\circ} \mathrm{C}, 16 \mathrm{~h}, 76 \%$; (5) oxalyl chloride, $\mathrm{Et}_{2} \mathrm{O}, \mathrm{rt}, 16 \mathrm{~h}$; (6) $\mathrm{NaH}_{2} \mathrm{PO}_{2} . \mathrm{H}_{2} \mathrm{O}, \mathrm{Pd} / \mathrm{C}, \mathrm{H}_{2} \mathrm{O} / 1$,4-dioxane (1:5), $100{ }^{\circ} \mathrm{C}, 72 \mathrm{~h}, 36 \%$ over two steps. (B) Isogenic strains with the indicated genotypes (mcm4+ (AW279), mcm4-AID (AW1893), mcm4-AID-V5 (AW1899), OsTIR1 ${ }^{\text {F74A }}$ (AW1703), mcm4-AID OsTIR1 ${ }^{\text {F74A }}$ (AW1925), mcm4-AID-V5 OsTIR1 ${ }^{\text {F74A }}$ (AW1937)) were spotted onto YEA plates and plates containing $10 \mathrm{nM} 5^{\prime}$ adamantyl-IAA (5'a-IAA). Plates were grown at indicated temperatures for 3 days. The experiment was repeated and similar results were obtained. (C) Representative immunoblot on total protein extract from mcm4+ (AW279), mcm4-AID-V5 (AW1899) and mcm4-AID-V5 OsTIR1 ${ }^{F 74 A}$ (AW1937) cells treated with $10 \mathrm{nM}$ 5'adamantyl-IAA (5'a-IAA) and sampled at the indicated time points and detected using anti-V5 antibody. Anti-tubulin was used as a loading control. (D) Graph of quantified anti-V5 signal normalised against anti-tubulin signal for (C) (data represented as the mean values +/ - SD of three independent experiments). (E) Isogenic strains with the indicated genotypes (rad52+ (AW279), rad52-AID (AW1901), rad52-AID-V5 (AW1907) OsTIR1 ${ }^{\text {F74A }}$ (AW1703), rad52-AID OsTIR1 ${ }^{\text {F74A }}$ (AW1962), rad52-AID-V5 OsTIR1 ${ }^{\text {F74A }}$ (AW1968), rad52 $($ AW1581)) were spotted onto YEA plates and plates containing $100 \mathrm{nM} 5^{\prime}$ adamantyl-IAA (5'a-IAA) and/or genotoxic agent hydroxyurea (HU) at the indicated concentrations. Plates were grown at $30{ }^{\circ} \mathrm{C}$ for 3 days. The experiment was repeated and similar results were obtained. (F) Representative immunoblot on total protein extract from rad52+ (AW279), rad52-AID-V5 (AW1907) and rad52-AID-V5 OsTIR1 ${ }^{\text {F74A }}$ (AW1968) cells treated with $100 \mathrm{nM} 5^{\prime}$ adamantyl-IAA (5'a-IAA) and sampled at indicated time points and detected using anti-V5 antibody. Anti-tubulin was used as a loading control. (G) Graph of quantified anti-V5 signal normalised against anti-tubulin signal for (F) (data represented as the mean values +/ - SD of three independent experiments).

Immunoblot analysis shows no auxin-independent depletion of Mcm4-AID-V5 in cells expressing OsTIR1 ${ }^{\text {F74A }}$ (Figure 3C,D) in contrast to $m c m 4-A I D-V 5$ OsTIR1 ${ }^{W T}$ cells (Figure 2B,C). Furthermore, Figure 3C,D show that the kinetics of depletion are faster in $m c m 4-A I D-V 5$ OsTIR $1^{\text {F74A }}$ cells after the addition of $5^{\prime}$ adamantyl-IAA (94\% reduction after $15 \mathrm{~min}$ ) when compared to $m c m 4-A I D-V 5$ OsTIR $1^{W T}$ cells treated with NAA (see Figure $2 \mathrm{~B}, \mathrm{C})(90 \%$ reduction after $15 \mathrm{~min})$. These faster kinetics may be due to the higher affinity of the $5^{\prime}$ adamantyl-IAA-induced OsTIR1 ${ }^{\text {F74A }}$ interaction when compared to the NAA-induced OsTIR $1^{\text {WT }}$ interaction [18]. Surprisingly, steady-state proteins levels after addition of $5^{\prime}$ adamantyl-IAA to $m c m 4-A I D-V 5$ OsTIR $1^{\text {F74A }}$ cells are similar to those seen for $m c m 4-A I D-V 5$ OsTIR $1^{W T}$ cells after NAA addition (approximately $4 \%$ of no TIR1 levels).

To test AID2 using Rad52, rad52-AID OsTIR1 ${ }^{\text {F74A }}$ and rad52-AID-V5 OsTIR1 ${ }^{\text {F74A }}$ cells were created. We tested a range of $5^{\prime}$ adamantyl-IAA concentrations $(1,000 \mathrm{nM}, 100 \mathrm{nM}$ and $10 \mathrm{nM}$ ) using spot test analysis of rad52-AID OsTIR1 ${ }^{F 74 A}$ cells (Figure S5B) and determined that a concentration of $100 \mathrm{nM} 5^{\prime}$ adamantyl-IAA was required to maximally induce the rad52 phenotype-a 500-fold lower concentration as compared to NAA. The Rad52AID/OsTIR1 ${ }^{\text {F74A }}$ system is also clearly more efficient than the Rad52-AID/OsTIR1 ${ }^{\text {WT }}$ system. Growth rates of rad52-AID OsTIR1 ${ }^{F 74 A}$ cells were reduced in the presence of 5'adamantyl-IAA compared to rad52-AID cells (Figure 3E), whereas rad52-AID OsTIR1 ${ }^{\text {WT }}$ cells treated with NAA do not show reduced growth rate (Figure 2D). Furthermore, rad52AID OsTIR1 ${ }^{\text {F74A }}$ cells treated with 5'adamantyl-IAA show a marked reduction in cell viability when grown in the presence of HU (Figure $3 \mathrm{E}$ ) when compared to rad52-AID OsTIR1 ${ }^{W T}$ cells treated with NAA (Figure 2D). However, despite improved efficiency of Rad52 degradation by AID2, rad52-AID/OsTIR $1^{\text {F74A }}$ cells treated with $5^{\prime}$ adamantyl-IAA are not as slow growing nor as sensitive to $H U$ as rad52 $\Delta$ cells, implying residuary Rad52 activity remains in these cells.

As seen for Mcm4-AID-V5, immunoblot analysis shows no auxin-independent depletion of Rad52-AID-V5 in cells expressing OsTIR1 ${ }^{\text {F74A }}$ (Figure 3F,G). Furthermore, Figure 3G shows that the kinetics of depletion are faster in rad52-AID-V5 OsTIR1 ${ }^{F 74 A}$ cells after the addition of $5^{\prime}$ adamantyl-IAA ( $94 \%$ reduction after $15 \mathrm{~min}$ ) when compared to rad52-AIDV5 OsTIR $1^{W T}$ cells treated with NAA (Figure $\left.2 \mathrm{~B}, \mathrm{C}\right)(77 \%$ reduction after $15 \mathrm{~min})$. The steady-state protein levels after addition of $5^{\prime}$ adamantyl-IAA to rad52-AID-V5 OsTIR1 ${ }^{F 74 A}$ cells are significantly lower than those seen for rad52-AID-V5 OsTIR1 ${ }^{\text {WT }}$ cells treated with NAA $(1.6 \%$ and $3.6 \%$, respectively), which explains the slower growth rates and increased HU sensitivity described above. 
As recently shown by others [6], the AID2 (OsTIR1 ${ }^{\mathrm{F74A}} / 5^{\prime}$ adamantyl-IAA) system is clearly an improvement on the AID (OsTIR $1^{\text {WT }}$ /NAA) system with faster degradation kinetics, much lower ligand concentrations and no auxin-independent depletion of AIDtagged proteins. However, despite this increase in functionality, observable levels of AID-tagged proteins remain.

\subsection{Use of DSR Elements Allows Generation of Stricter S. pombe Mutants}

Despite significantly improving the fission yeast auxin-dependent degron system, the reduction in protein levels was insufficient to obtain a conditional rad52-null mutant phenotype. To achieve such a phenotype, other levels of regulation are therefore required. The regulation of transcription is an effective and well-defined option but requires additional genome editing with the insertion of a regulatable promoter sequence and the manipulation of the growth media in order to downregulate transcription. Determinant of Selective Removal (DSR) elements have previously been shown to effectively modulate protein levels [23]. As DSR elements are usually located at the $3^{\prime}$ end of the transcript they can easily be incorporated into the AID-plasmid sequence (Figure 1E) and therefore require no extra steps. The use of DSRs does, however, have the disadvantage that protein levels are constitutively lowered.

Using the DSR containing AID-plasmids outlined in Figure 1E, we C-terminally tagged the rad52 locus with AID-spo5DSR, AID-eDSR, AID-V5-spo5DSR and AID-V5eDSR. To determine the relative effectiveness of the spo5DSR and eDSR on Rad52-AID$\mathrm{V} 5$ levels, Western blot analysis was performed. Immunoblot of total protein extracted from logarithmically growing rad52-AID (NO DSR), rad52-AID-spo5DSR and rad52-AID$e D S R$ cells shows the effect of DSR elements on constitutively reducing protein levels (Figure 4A). The previously untested eDSR element-a mutant form of spo5DSR engineered to contain two additional copies of the DSRcore element-is more effective at reducing levels than spo5DSR. Quantification of Rad52-AID-V5 proteins levels revealed that the spo5DSR element reduces the level on average by $84 \%$ and the eDSR element by $98 \%$ (Figure 4B). As above, addition of 5'adamantyl-IAA to rad52-AID-spo5DSR and rad52AID-eDSR cells exogenously expressing OsTIR $1^{\mathrm{F} 74 \mathrm{~A}}$ caused rapid depletion of Rad52-AIDtagged proteins (Figure 4C,D, respectively). Interestingly, in rad52-AID-V5-spo5DSR cells treated with $5^{\prime}$ adamantyl-IAA, steady-state protein levels were still detectable despite a reduction in initial protein levels by $84 \%$ (Figure $4 \mathrm{~B}, \mathrm{C}$ ). This suggests that as the AIDtagged protein level is reduced, the efficiency of degradation is also reduced. However, in rad52-AID-V5-eDSR cells treated with 5'adamantyl-IAA, where initial levels are reduced by $98 \%$, steady-state protein levels were un-detectable (Figure $4 \mathrm{D}$ ).

The effects of the reduction in Rad52-AID protein levels due to the DSR elements are revealed by spot test analysis (Figure 4E). Predictably, as levels of Rad52 decrease in rad52-AID (NO DSR), rad52-AID-spo5DSR and rad52-AID-eDSR cells, sensitivity to $4 \mathrm{mM}$ $\mathrm{HU}$ increases. Interestingly, HU sensitivity was slightly increased when a V5 tag for each of these strains was also present, implying that the V5 tag reduces Rad52 activity (Figure 4E). In the presence of $100 \mathrm{nM} 5$ 'adamantyl-IAA, rad52-AID-spo5DSR OsTIR1 ${ }^{\text {F74A }}$ cells exhibit a slow growth phenotype that is significantly more pronounced as seen for rad52-AID/OsTIR $1^{F 74 A}$ cells, but not as slow growing as rad52 $\Delta$ cells (Figure $4 \mathrm{E}$ ). This pattern is repeated for plates supplemented with HU. rad52-AID-spo5DSR OsTIR1 ${ }^{F 74 A}$ cells exhibit sensitivity to HU that is significantly more severe than that seen for rad52-AID OsTIR1 ${ }^{F 74 A}$ cells but not as sensitive as rad52 $\Delta$ cells. Unexpectedly, when grown in the presence of 5'adamantyl-IAA, rad52-AID-eDSR OsTIR1 $1^{F 74 A}$ cells are as slow growing and as sensitive to $\mathrm{HU}$ as rad52-AID-spo5DSR OsTIR1 ${ }^{\text {F74A }}$ cells. This was surprising as the level of Rad52-AID-V5 protein is clearly lower when associated with eDSR than that of Rad52-AID-V5 associated with spo5DSR (Figure 4C,D, respectively). This may reflect the sensitivity limit of the spot test assay. 

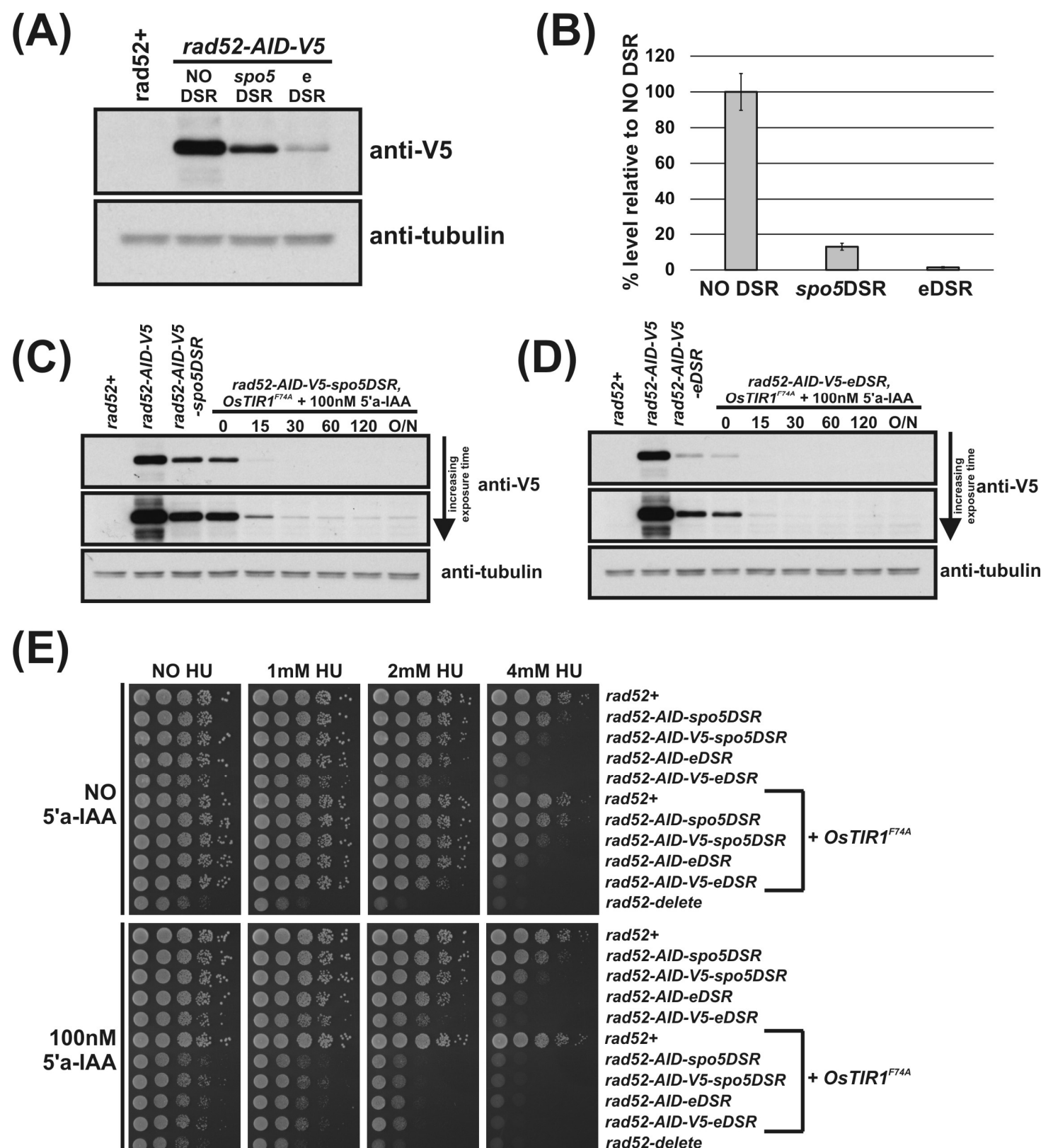

Figure 4. DSR elements improve AID2/OsTIR1 ${ }^{\mathrm{F} 74 \mathrm{~A}}$. (A) Representative immunoblot on total protein extract from rad52+ (AW279), rad52-AID-V5 (NO DSR) (AW1907), rad52-AID-V5-spo5DSR (spo5DSR) (AW1921) and rad52-AID-V5-eDSR (eDSR) (AW2017) cells detected using anti-V5 antibody. Anti-tubulin was used as a loading control. (B) Histogram of quantified antiV5 signal normalised against anti-tubulin signal for (A) (data represented as the mean values +/ - SD of three independent experiments). (C) Representative immunoblot on total protein extract from control and rad52-AID-V5-spo5DSR OsTIR1 ${ }^{\text {F74A }}$ (AW1976) cells treated with $100 \mathrm{nM}$ 5'adamantyl-IAA (5'a-IAA) and sampled at indicated time points and detected using anti-V5 antibody. Anti-tubulin was used as a loading control. (D) Representative immunoblot on total protein extract from control and rad52-AID-V5-eDSR OsTIR1 ${ }^{F 74 A}$ (AW2026) cells treated with $100 \mathrm{nM} 5^{\prime}$ adamantyl-IAA (5'a-IAA) and sampled at the indicated time points and detected using anti-V5 antibody. Anti-tubulin was used as a loading control. (E) Isogenic strains with indicated genotypes (rad52+ (AW279), rad52-AID-spo5DSR (AW1915), rad52-AID-V5-spo5DSR (AW1921), rad52AID-eDSR (AW2011), rad52-AID-V5-eDSR (AW2017), OsTIR1 ${ }^{F 74 A}$ (AW1703), rad52-AID-spo5DSR) OsTIR1 ${ }^{\text {F74A }}$ (AW1970), rad52-AID-V5-spo5DSR OsTIR1 ${ }^{\text {F74A }}$ (AW1976), rad52-AID-eDSR OsTIR1 ${ }^{\text {F74A }}$ (AW2024), rad52-AID-V5-eDSR OsTIR1 F74A (AW2026), rad52 $\Delta$ (AW1581)) were spotted onto YEA plates and plates containing $100 \mathrm{nM} 5^{\prime}$ adamantyl-IAA (5' a-IAA) and/or genotoxic agent hydroxyurea (HU) at the indicated concentrations. Plates were grown at $30{ }^{\circ} \mathrm{C}$ for 3 days. Experiment was repeated and similar results obtained. 
The use of DSR elements clearly reduces AID-target protein levels leading to a more penetrative phenotype. Frustratingly, despite the higher efficiency of the eDSR element compared to spo5DSR, rad52-AID-eDSR OsTIR1 ${ }^{\text {F74A }}$ and rad52-AID-spo5DSR OsTIR1 ${ }^{\text {F74A }}$ are as equally sensitive to HU when $5^{\prime}$ adamantyl-IAA is present (Figure 4E) and neither are as sensitive as rad52 $\Delta$ cells. However, the residual level of Rad52 is very low.

\section{Discussion}

Here, we describe the construction of a powerful protein-depletion system in S. pombe based upon the auxin-inducible degron (AID) system. We created two sets of plasmid vectors for this study. The first is a series of stable integration expression vectors that target the $S$. pombe arg3-D4 locus (Figure S1). The vectors include the arg3 gene sequence that is deleted at the $\arg 3-D 4$ locus, a range of $S$. pombe adh1 gene promoter sequences of varying strengths and, to clone the gene to be expressed, an MCS. The plasmid vectors are designed to integrate a single stable copy at the $\arg 3-D 4$ locus restoring arginine prototrophy.

The second set of plasmids are to fuse the AID tag to the C-terminus of the target protein (Figure 1E). The AID constructs include a flexible ten amino acid linker, the fulllength $A$. thaliana IAA17 AID tag (a 229-amino-acid peptide) and, for the detection of AIDtagged proteins by immunoblot, a series of common epitope tags for which commercial antibodies are available. Also included is the option of one of two 'Determinants of Selective Removal' (DSR) elements: one derived from the S. pombe spo5 gene and an improved, mutated version of the spo5DSR termed eDSR. DSR elements usually prevent the accumulation of meiosis-specific mRNAs during the mitotic cell cycle in fission yeast by targeting them for destruction by nuclear exosomes. Here, they result in the constitutive reduction in AID-tagged protein levels. The AID constructs can be inserted into the genome using Cre recombinase-mediated cassette exchange [27] or by using a standard PCR-mediated tagging procedure commonly used for fission yeast.

Using the integration vectors, we successfully inserted at the arg3-D4 locus sequences to express the auxin receptor TIR1 protein from $O$. sativa (OsTIR $1^{\mathrm{WT}}$ ) and an auxin-binding site mutant of OsTIR1, OsTIR1 ${ }^{\text {F74A }}$. Importantly, both forms of OsTIR1 were not fused to Skp1. Overexpression of either OsTIR1 ${ }^{\mathrm{WT}}$ or OsTIR $1^{\mathrm{F} 74 \mathrm{~A}}$ from the strong constitutive adh1 promoter had no observable effect on cell growth. OsTIR1 ${ }^{\text {F74A }}$ binds the auxin analog 5'adamantyl-IAA with a higher affinity than OsTIR1 and natural auxin [18]. This synthetic pairing forms the basis of AID version 2 (AID2) the equivalent of which has recently been shown to perform better than AID in chicken DT40 cells, budding yeast, mammalian cells and mice [6,19].

Both AID and AID2 produced a conditional Mcm 4 null phenotype at $30^{\circ} \mathrm{C}$. However, as predicted, AID2 required much lower ligand concentration (5,000-fold less), showed slightly faster degradation kinetics and no auxin-independent proteolysis of Mcm4 or Rad52 as compared with AID. The effective concentration of 5-adamaltyl-IAA was higher for Rad52 (100 nM) than for Mcm4 (10 nM). This was surprising considering quantitative analysis of the fission yeast proteomes has shown that in vegetatively growing cells there are approximately 3-fold more Mcm4 protein molecules than Rad52 protein molecules [38] and Nishimura et al. [19] have demonstrated that depletion of AID-tagged CENP-H by 5'-adamantyl-IAA is dose dependent. The AID system may therefore be intrinsically better suited to regulating essential proteins: below a critical threshold level, the cell becomes inviable and a null phenotype achieved. Despite the higher ligand concentration, a lower steady-state protein level and the use of highly effective DSR elements, residual Rad52 activity remains after depletion (Figure 4C) (due possibly to TIR1 being limiting). Tandem arrays of functional truncated forms of the AID tag have been used to dramatically increase the efficiency of previous AID systems (termed '3XminiAID'; $[39,40]$ ). Use of a 3XminiAID-style tag may be sufficient to achieve a conditional-null Rad52 phenotype and we are currently testing this. However, anecdotal evidence from our lab suggests that fusion of the 3 Xmini-AID tag to the target protein either partially or completely removes protein function more often than with full-length AID. 
Despite no conditional null phenotype for Rad52 being achieved using either AID system, AID2 performed better than AID. The use of DSR elements successfully improved the severity of the conditional mutant phenotype, but a very low level of Rad52 activity remains. Despite the eDSR reducing initial protein levels by $94 \%$ and spo5DSR by $74 \%$ and immunoblots showing less Rad52-AID in rad52-AID-eDSR cells treated with $5^{\prime}$ adamantylIAA than rad52-AID-spo5DSR cells treated with 5'adamantyl-IAA, rad52-AID-eDSR and rad52-AID-spo5DSR cells surprisingly exhibited the same level of HU sensitivity.

A detailed protocol for the synthesis of $5^{\prime}$ adamantyl-IAA is provided in the materials and methods section. In addition, $5^{\prime}$ adamantyl-IAA is now commercially available (https:/ / www.tcichemicals.com/GB/en/p/A3390 (accessed on 6 June 2021)). We found 5 'adamantyl-IAA to be more chemically stable than NAA. We incubated an aliquot of 5 'adamantyl-IAA at room temperature for 7 days and, when tested, we observed no loss in activity (Figure S4). In our hands, NAA was found to be chemically labile with freeze/thawing of frozen stocks reducing activity. Only freshly prepared NAA stocks were used.

\section{Conclusions}

Overall, we have significantly improved the utility of the auxin-inducible degron system in S. pombe and this should enhance the study of gene function in vivo by more effectively reducing the levels of gene product. As auxin response has been shown to be intrinsically linked to cellular TIR1 concentration $[10,32,33]$, the increase in TIR1 expression level is the probable reason for the concomitant increase in AID efficiency. The relative thermostability of OsTIR1 compared to AtTIR1 [1] also means that our new system works also more efficiently at the higher temperatures of 30 and $36^{\circ} \mathrm{C}$.

The OsTIR system was further enhanced by introducing the synthetic pairing of the binding-site mutant OsTIR1 ${ }^{\mathrm{F} 74 \mathrm{~A}}$ with the auxin analogue $5^{\prime}$ adamantyl-IAA (AID2). Because neither the AID or AID2 systems abolish AID-tagged protein levels completely, we also introduced the ability to regulate the initial protein levels by introducing a DSR sequence that restricts the mRNA level and thus reduces the starting protein level. This may be particularly helpful when it is desirable to reduce gene function as much as possible to approach a null phenotype. While both the AID and AID2 systems are highly effective for regulating essential proteins to achieve a null-like phenotype, as seen for Mcm4, we note that residual activity remains when assessing a non-essential gene for function, even when combining AID2 with DSR elements.

Supplementary Materials: The following are available online at https:/ / www.mdpi.com/article/10 .3390/genes12060882/s1, Figure S1: Overview of the arg3-D4 integration expression vector system. (a) Sequence of the arg3-D4 locus [24]. Underlined capital letters: pBR322 plasmid sequence; sequence highlighted in yellow: $\arg 3-D 4$ homology used in construction of $\arg 3+$ expression vectors (see b). (b) Schematic illustration showing arg3-D4 integration expression vectors. HOM: the two homology sequences used to insert the linear expression construct at the arg3-D4 locus (see a); MCS: multiple clone site; number in parenthesis after plasmid name: Addgene ID. (c) Schematic illustration showing integration of $P_{a d h 1}-O S T I R 1^{W T}-N L S-T_{A D H 1}$ and $P_{a d h 1}-O s T I R 1^{F 74 A}-N L S-T_{A D H 1}$ expression constructs at $\arg 3-D 4$ locus using arg3-D4 integration expression vector system; cmb1: gene neighbouring $\arg 3$. Figure S2: The spo5DSR and eDSR elements. (a) The 157bp DSR element from the S. pombe spo5 gene downstream region as identified by Harigaya et al. [20]. Sequences identical to core hexanucleotide $\mathrm{U}(\mathrm{U} / \mathrm{C}) \mathrm{AAAC}$ motifs are highlighted. Boxed sequences are those identified as similar to the core motif. (b) The $158 \mathrm{bp}$ eDSR sequence derived from the spo5DSR element. Underlined sequences are additional core TTAAAC motifs (mutated bases shown in lower case). Figure S3: Alignment of Oryza sativa transport inhibitor response protein 1 (OsTIR1) and Arabidopsis thaliana transport inhibitor response protein 1 (AtTIR1) proteins. AtTIR1 (GeneID 825473) and OsTIR1 (GeneID 4335696) were aligned using the ClustalW software. Black and grey boxes indicate identical and similar amino acids, respectively. Position F79 of AtTIR1 and the corresponding F74 of OsTIR1 are arrowed. Figure S4: 5'adamantyl-IAA is chemically stable. Isogenic strains with indicated genotypes (rad52+ (AW279), rad52-HA-AID, OsTIR1 ${ }^{\text {F74A }}$ (AW1680), rad52-HA-AID OsTIR1 ${ }^{\text {F74A }}$ (AW1681), rad52-HA-AID- 
spo5DSR OsTIR1 ${ }^{\text {F74A }}$ (AW1682), rad52-HA-AID-spo5DSR OsTIR1 ${ }^{\text {F74A }}$ (AW1683), rad52 $(\mathrm{AW} 1581)$ ) were spotted onto YEA plates and plates containing $100 \mathrm{nM}$ 5'adamantyl-IAA (5'a-IAA) and/or genotoxic agent hydroxyurea (HU) at the indicated concentrations. 5'adamantyl-IAA added to plates from frozen stock $\left(-20^{\circ} \mathrm{C}\right.$ stock) or from an aliquot incubated at room temperature for 7 days (7d RT). Plates were grown at $30^{\circ} \mathrm{C}$ for 3 days, Figure S5: Titration of 5'adamantyl-IAA to determine effective ligand concentration. (a) Mcm4-AID. Isogenic strains with indicated genotypes (mcm4+ (AW279), mcm4-AID (AW1893), OsTIR1 ${ }^{\text {F74A }}$ (AW1703), mcm4-AID OsTIR1 ${ }^{\text {F74A }}$ (AW1925)) were spotted onto YEA plates and plates containing $100 \mathrm{nM}$ 5'adamantyl-IAA (5'a-IAA), $10 \mathrm{nM}$ 5'adamantyl-IAA (5'a-IAA) and $1 \mathrm{nM}$ 5'adamantyl-IAA (5'a-IAA). Plates were grown at $30^{\circ} \mathrm{C}$ for 3 days. (b) Rad52AID. Isogenic strains with indicated genotypes (rad52+ (AW279), rad52-AID (AW1901), OsTIR1 ${ }^{F 74 A}$ (AW1703), rad52-AID OsTIR1 ${ }^{\text {F74A }}$ (AW1962), rad52 (AW1581)) were spotted onto YEA plates and plates containing $1000 \mathrm{nM}$ 5'adamantyl-IAA (5'a-IAA), $100 \mathrm{nM}$ 5'adamantyl-IAA (5'a-IAA) and 10 nM 5'adamantyl-IAA (5'a-IAA). Plates were grown at $30^{\circ} \mathrm{C}$ for 3 days. Note: each panel in (b) is a composite of two images (both taken of the same plate) excising irrelevant strains between rad52-AID and rad52-delete.

Author Contributions: Conceptualisation, A.M.C. and A.T.W.; methodology, A.T.W. and S.H.-H.; validation, A.T.W. and S.H.-H.; formal analysis, A.M.C., A.T.W., S.H.-H. and J.S.; investigation, A.T.W. and S.H.-H.; data curation, A.T.W. and S.H.-H.; writing-original draft preparation, A.T.W.; writing-review and editing, A.M.C., A.T.W., S.H.-H. and J.S.; funding acquisition, A.M.C. and J.S. All authors have read and agreed to the published version of the manuscript.

Funding: This research was funded in part by the Wellcome Trust [110047/Z/15/Z]. S.H-H. and J.S. were funded by EPSRC grant number EP/P026990/1. For the purpose of Open Access, the author has applied a CC BY public copyright licence to any Author Accepted Manuscript version arising from this submission.

Data Availability Statement: Primary data are available from the authors on request.

Acknowledgments: We thank Li-Lin Du for kindly supplying an initial aliquot of 5 'adamantyl-IAA.

Conflicts of Interest: The authors declare no conflict of interest. The funders had no role in the design of the study; in the collection, analyses, or interpretation of data; in the writing of the manuscript, or in the decision to publish the results.

\section{References}

1. Nishimura, K.; Fukagawa, T.; Takisawa, H.; Kakimoto, T.; Kanemaki, M. An auxin-based degron system for the rapid depletion of proteins in nonplant cells. Nat. Methods 2009, 6, 917-922. [CrossRef]

2. Teale, W.D.; Paponov, I.A.; Palme, K. Auxin in action: Signalling, transport and the control of plant growth and development. Nat. Rev. Mol. Cell Biol. 2006, 7, 847-859. [CrossRef]

3. Costa, E.A.; Subramanian, K.; Nunnari, J.; Weissman, J.S. Defining the physiological role of SRP in protein-targeting efficiency and specificity. Science 2018, 359, 689-692. [CrossRef]

4. Nora, E.P.; Goloborodko, A.; Valton, A.L.; Gibcus, J.H.; Uebersohn, A.; Abdennur, N.; Dekker, J.; Mirny, L.A.; Bruneau, B.G. Targeted Degradation of CTCF Decouples Local Insulation of Chromosome Domains from Genomic Compartmentalization. Cell 2017, 169, 930-944. [CrossRef]

5. Muhar, M.; Ebert, A.; Neumann, T.; Umkehrer, C.; Jude, J.; Wieshofer, C.; Rescheneder, P.; Lipp, J.J.; Herzog, V.A.; Reichholf, B.; et al. SLAM-seq defines direct gene-regulatory functions of the BRD4-MYC axis. Science 2018, 360, 800-805. [CrossRef] [PubMed]

6. Yesbolatova, A.; Saito, Y.; Kitamoto, N.; Makino-Itou, H.; Ajima, R.; Nakano, R.; Nakaoka, H.; Fukui, K.; Gamo, K.; Tominari, Y.; et al. The auxin-inducible degron 2 technology provides sharp degradation control in yeast, mammalian cells, and mice. Nat. Commun. 2020, 11, 5701. [CrossRef] [PubMed]

7. Zhang, L.; Ward, J.D.; Cheng, Z.; Dernburg, A.F. The auxin-inducible degradation (AID) system enables versatile conditional protein depletion in C. elegans. Development 2015, 142, 4374-4384. [CrossRef] [PubMed]

8. Bence, M.; Jankovics, F.; Lukácsovich, T.; Erdélyi, M. Combining the auxin-inducible degradation system with CRISPR/Cas9based genome editing for the conditional depletion of endogenous Drosophila melanogaster proteins. FEBS J. 2017, 284, 1056-1069. [CrossRef] [PubMed]

9. Kleinjan, D.A.; Wardrope, C.; Nga Sou, S.; Rosser, S.J. Drug-tunable multidimensional synthetic gene control using inducible degron-tagged dCas9 effectors. Nat. Commun. 2017, 8, 1191. [CrossRef]

10. Kanke, M.; Nishimura, K.; Kanemaki, M.; Kakimoto, T.; Takahashi, T.S.; Nakagawa, T.; Masukata, H. Auxin-inducible protein depletion system in fission yeast. BMC Cell Biol. 2011, 12, 1-6. [CrossRef]

11. Yamagishi, Y.; Sakuno, T.; Shimura, M.; Watanabe, Y. Heterochromatin links to centromeric protection by recruiting shugoshin. Nature 2008, 455, 251-255. [CrossRef] 
12. Kanke, M.; Kodama, Y.; Takahashi, T.S.; Nakagawa, T.; Masukata, H. Mcm10 plays an essential role in origin DNA unwinding after loading of the CMG components. EMBO J. 2012, 31, 2182-2194. [CrossRef] [PubMed]

13. Handa, T.; Kanke, M.; Takahashi, T.S.; Nakagawa, T.; Masukata, H. DNA polymerization-independent functions of DNA polymerase epsilon in assembly and progression of the replisome in fission yeast. Mol. Biol. Cell 2012, 23, 3240-3253. [CrossRef]

14. Kakui, Y.; Rabinowitz, A.; Barry, D.J.; Uhlmann, F. Condensin-mediated remodeling of the mitotic chromatin landscape in fission yeast. Nat. Genet. 2017, 49, 1553-1557. [CrossRef] [PubMed]

15. Moiseeva, V.; Amelina, H.; Collopy, L.C.; Armstrong, C.A.; Pearson, S.R.; Tomita, K. The telomere bouquet facilitates meiotic prophase progression and exit in fission yeast. Cell Discov. 2017, 3, 17041. [CrossRef] [PubMed]

16. Sathyan, K.M.; McKenna, B.D.; Anderson, W.D.; Duarte, F.M.; Core, L.; Guertin, M.J. An improved auxin-inducible degron system preserves native protein levels and enables rapid and specific protein depletion. Genes Dev. 2019, 33, 1441-1455. [CrossRef] [PubMed]

17. Uchida, N.; Takahashi, K.; Iwasaki, R.; Yamada, R.; Yoshimura, M.; Endo, T.A.; Kimura, S.; Zhang, H.; Nomoto, M.; Tada, Y.; et al. Chemical hijacking of auxin signaling with an engineered auxin-TIR1 pair. Nat. Chem. Biol. 2018, 14, 299-305. [CrossRef]

18. Yamada, R.; Murai, K.; Uchida, N.; Takahashi, K.; Iwasaki, R.; Tada, Y.; Kinoshita, T.; Itami, K.; Torii, K.U.; Hagihara, S. A Super Strong Engineered Auxin-TIR1 Pair. Plant Cell Physiol. 2018, 59, 1538-1544. [CrossRef]

19. Nishimura, K.; Yamada, R.; Hagihara, S.; Iwasaki, R.; Uchida, N.; Kamura, T.; Takahashi, K.; Torii, K.U.; Fukagawa, T. A super-sensitive auxin-inducible degron system with an engineered auxin-TIR1 pair. Nucleic Acids Res. 2020, 48, e108. [CrossRef] [PubMed]

20. Harigaya, Y.; Tanaka, H.; Yamanaka, S.; Tanaka, K.; Watanabe, Y.; Tsutsumi, C.; Chikashige, Y.; Hiraoka, Y.; Yamashita, A.; Yamamoto, M. Selective elimination of messenger RNA prevents an incidence of untimely meiosis. Nature 2006, 442, 45-50. [CrossRef]

21. Yamanaka, S.; Yamashita, A.; Harigaya, Y.; Iwata, R.; Yamamoto, M. Importance of polyadenylation in the selective elimination of meiotic mRNAs in growing S. pombe cells. EMBO J. 2010, 29, 2173-2181. [CrossRef]

22. Yamashita, A.; Shichino, Y.; Tanaka, H.; Hiriart, E.; Touat-Todeschini, L.; Vavasseur, A.; Ding, D.Q.; Hiraoka, Y.; Verdel, A.; Yamamoto, M. Hexanucleotide motifs mediate recruitment of the RNA elimination machinery to silent meiotic genes. Open Biol. 2012, 2, 120014. [CrossRef]

23. Watson, A.T.; Daigaku, Y.; Mohebi, S.; Etheridge, T.J.; Chahwan, C.; Murray, J.M.; Carr, A.M. Optimisation of the Schizosaccharomyces pombe urg1 expression system. PLOS ONE 2013, 8, e83800. [CrossRef]

24. Waddell, S.; Jenkins, J.R. arg3+, a new selection marker system for Schizosaccharomyces pombe: Application of ura4+ as a removable integration marker. Nucleic Acids Res. 1995, 23, 1836-1837. [CrossRef] [PubMed]

25. Moreno, S.; Klar, A.; Nurse, P. Molecular Genetic Analysis of Fission Yeast Schizosaccharomyces Pombe; Guide to Yeast Genetics and Molecular Biology, Methods in Enzymology; Elsevier: Amsterdam, Netherlands, 1991; pp. 795-823.

26. Zach, R.; Carr, A.M. Increased expression of Polठ does not alter the canonical replication program in vivo. Wellcome Open Res. 2021, 6, 44. [CrossRef]

27. Watson, A.T.; Garcia, V.; Bone, N.; Carr, A.M.; Armstrong, J. Gene tagging and gene replacement using recombinase-mediated cassette exchange in Schizosaccharomyces pombe. Gene 2008, 407, 63-74. [CrossRef] [PubMed]

28. Bähler, J.; Wu, J.Q.; Longtine, M.S.; Shah, N.G.; McKenzie, A.; Steever, A.B.; Wach, A.; Philippsen, P.; Pringle, J.R. Heterologous modules for efficient and versatile PCR-based gene targeting in Schizosaccharomyces pombe. Yeast 1998, 14, 943-951. [CrossRef]

29. Hentges, P.; Van Driessche, B.; Tafforeau, L.; Vandenhaute, J.; Carr, A.M. Three novel antibiotic marker cassettes for gene disruption and marker switching in Schizosaccharomyces pombe. Yeast 2005, 22, 1013-1019. [CrossRef]

30. Watson, A.T.; Werler, P.; Carr, A.M. Regulation of gene expression at the fission yeast Schizosaccharomyces pombe urg1 locus. Gene 2011, 484, 75-85. [CrossRef] [PubMed]

31. Yokobayashi, S.; Watanabe, Y. The kinetochore protein Moa1 enables cohesion-mediated monopolar attachment at meiosis I. Cell 2005, 123, 803-817. [CrossRef]

32. Gray, W.M.; del Pozo, J.C.; Walker, L.; Hobbie, L.; Risseeuw, E.; Banks, T.; Crosby, W.L.; Yang, M.; Ma, H.; Estelle, M. Identification of an SCF ubiquitin-ligase complex required for auxin response in Arabidopsis thaliana. Genes Dev. 1999, 13, 1678-1691. [CrossRef] [PubMed]

33. Maraschin, F.S.; Memelink, J.; Offringa, R. Auxin-induced, SCF(TIR1)-mediated poly-ubiquitination marks AUX/IAA proteins for degradation. Plant J. 2009, 59, 100-109. [CrossRef] [PubMed]

34. Morawska, M.; Ulrich, H.D. An expanded tool kit for the auxin-inducible degron system in budding yeast. Yeast 2013, 30, 341-351. [CrossRef] [PubMed]

35. Nishimura, K.; Fukagawa, T. An efficient method to generate conditional knockout cell lines for essential genes by combination of auxin-inducible degron tag and CRISPR/Cas9. Chromosome Res. 2017, 25, 253-260. [CrossRef]

36. Zasadzińska, E.; Huang, J.; Bailey, A.O.; Guo, L.Y.; Lee, N.S.; Srivastava, S.; Wong, K.A.; French, B.T.; Black, B.E.; Foltz, D.R. Inheritance of CENP-A Nucleosomes during DNA Replication Requires HJURP. Dev. Cell 2018, 47, 348-362.e7. [CrossRef]

37. Doe, C.L.; Osman, F.; Dixon, J.; Whitby, M.C. DNA repair by a Rad22-Mus81-dependent pathway that is independent of Rhp51. Nucleic Acids Res. 2004, 32, 5570-5581. [CrossRef]

38. Marguerat, S.; Schmidt, A.; Codlin, S.; Chen, W.; Aebersold, R.; Bähler, J. Quantitative analysis of fission yeast transcriptomes and proteomes in proliferating and quiescent cells. Cell 2012, 151, 671-683. [CrossRef]

39. Kubota, T.; Nishimura, K.; Kanemaki, M.T.; Donaldson, A.D. The Elg1 replication factor C-like complex functions in PCNA unloading during DNA replication. Mol. Cell 2013, 50, 273-280. [CrossRef] 
40. Tanaka, S.; Miyazawa-Onami, M.; Iida, T.; Araki, H. iAID: An improved auxin-inducible degron system for the construction of a 'tight' conditional mutant in the budding yeast Saccharomyces cerevisiae. Yeast 2015, 32, 567-581. [CrossRef] 